\title{
Physiologische und ökologische Aspekte des Lebens in Ästuarien ${ }^{1,2}$
}

\author{
OTTO Kinne \\ Biologische Anstalt Helgoland, Zentrale, Hamburg-Altona
}

\begin{abstract}
Physiological and ecological aspects of life in estuaries. In spite of their structural diversity, estuarine organisms exhibit close functional similarities, particularly with respect to the compensatory measures employed to counteract the ill effects of their rough osmotic and thermal environment. Four groups of such compensatory possibilities are distinguished: escape, reduction of contact, regulation and adaptation. It should be emphasized that these compensation types may occur simultaneously in one and the same individual and that they may not always be distinguishable to the last detail. Nevertheless, such a subdivision makes it possible to present the multiplicity of physiological and ecological responses in some order and may prove a useful tool for further analyses. With respect to compensations for the potential ill effects of salinity, escape, reduction of contact and regulation (ion-, volume-and osmoregulation) appear to be most important. In regard to extreme temperature conditions, escape and adaptation represent the primary means for compensation. The information on hand indicates that compensations for adverse salinities are more immediately available and better developed than those for adverse temperatures. Unsuitable temperature conditions are tolerated rather than compensated for and thus may greatly affect metabolic rate, activity, reproduction and other functions as well as structural aspects. The most important characteristic of estuarine organisms - their euryplasticity seems to be a rather rare asset both in the plant and animal kingdom and phylogenetically quite conservative.
\end{abstract}

\section{EINLEITUNG}

Astuarien oder Flußmündungsgebiete sind Lebensräume mit unwirschen, recht speziellen Existenzbedingungen. Sie sind Übergangszonen zwischen den benachbarten, stabileren Meeres- und Süßwasserhabitaten, von denen sie sich insbesondere durch verstärkte Gradienten und Fluktuationen abiotischer und teilweise auch biotischer Umweltfaktoren unterscheiden. Ein Asstuar enthält Wasser, dessen mariner Anteil in verschiedenem Ausmaß verdünnt und modifiziert wird durch Flußwasser. Die wichtigsten Faktorenkomplexe, welche die Lebensbedingungen in einem Astuar bestimmen, sind Volumen und Beschaffenheit des Flußwassers, Gezeiten (Wasseraustausch) und Geomorphologie des Flußmündungsbereiches.

${ }^{1}$ Herm Professor Dr. Adolf Bückmann zum 65. Geburtstag am 17. 1. 1965 in Verehrung gewidmet.

${ }_{2}^{2}$ Ins Deutsche übertragene, veränderte Fassung eines Vortrages, welcher am 1. April 1964 auf Jekyll Island, Georgia (USA), gehalten worden ist. 
Diese generellen Aussagen treffen im Prinzip für alle Flußmündungsgebiete zu, auch wenn sich im einzelnen verschiedene Ästuar-Typen unterscheiden lassen. Sie charakterisieren ein Astuarium als ein Stress-Habitat, welches seinen permanenten Bewohnern ebenso wie den temporären Einwanderern (Besuchern) von Meer und Süßwasser physiologisch viel abverlangt und sie aufs höchste beansprucht. Permanente Ästuarbewohner erweisen sich in ihren physiologischen und ökologischen Reaktionen durchweg als ausgesprochen euryplastisch und sehr resistent gegen die vielfältigen Unbilden ihres rauhen Umweltklimas; sie haben ein großes "abiotisches Potential“. Ihr "biotisches Potential" (Widerstandsfähigkeit gegen biotische Faktoren; Konkurrenzvermögen) dagegen ist vielfach gering. Zahlreiche ästuarine Organismen sind offenbar durch Konkurrenten, Feinde oder Parasiten aus den klimatisch günstigeren Bedingungen im Meer oder Süßwasser vertrieben worden. Für diese Organismen repräsentiert das Ǎstuarium trotz seiner suboptimalen oder gar subnormalen physiko-chemischen Existenzbedingungen einen Zufluchtsort - ein Refugium, in dem sie Schutz finden vor anderen, sie bedrängenden Lebensformen, welche ihrerseits über ein geringes abiotisches Potential verfügen und daher auf Gebiete mit weniger extremen klimatischen Verhältnissen beschränkt bleiben. Einige Bewohner oder Besucher der Ästuarien frnden im Flußmündungsbereich möglicherweise auch günstigere Ernährungsbedingungen. Auf jeden Fall repräsentieren die meisten Astuarien Gebiete mit reduzierter interspezifischer Konkurrenz, in denen Verbreitung, Abundanz und Populationsdynamik primär von den abiotischen Faktoren abhängen.

Pro Raumeinheit ist die Artenzahl in Astuarien meist signifikant geringer als im benachbarten Meer oder Süßwasser. Die Individuenzahl dagegen kann sehr hoch sein, vor allem dann, wenn der Flußmündungsbereich eutroph ist. Besonders in den Tropen und Subtropen erreicht die Produktion lebender Substanz in eutrophen Astuarien vielfach erstaunlich hohe Werte. Dem Astuarium kommt dann häufig eine wichtige Bedeutung zu als Aufwuchs- und Futterplatz für eurysaline Besucher aus Meer- und Süßwasser.

Eine Analyse der speziellen physiologischen und ökologischen Eigenarten und Reaktionsmuster ästuariner Organismen ist äußerst diffizil - nicht zuletzt wegen der großen Zahl wirksamer Umweltqualitäten. Für die Reaktionen und das ökologische Verhalten eines Individuums ist letztlich eine Faktorenhierarchie verantwortlich, wobei sich die Einzelfaktoren zudem noch in ihren biologischen Wirkungen gegenseitig beeinflussen können. Zum gegenwärtigen Zeitpunkt ist es nicht einmal möglich, die wichtigsten Umweltqualitäten in ausreichendem Maße zu erfassen. Aus diesem. Grunde und weil unsere Kenntnisse über die physiologische Okologie ästuariner Organismen noch recht fragmentarisch sind, beschränke ich mich in der nachfolgenden Abhandlung auf zwei Umweltfaktoren: Salinität und Temperatur. Zusammen mit anderen Umweltqualitäten, wie Wasserbeschaffenheit, Wasserbewegung, Licht, Trïbung, gelöste Gase, Nährstoffe und Substratbeschaffenheit, sind diese beiden Faktoren von besonderer physiologischer und ökologischer Bedeutung. Ästuarien enthalten eine Anhäufung von Genotypen mit ähnlichen physiologisch-ökologischen Eigenarten. Es ist meine Absicht, diese spezifischen Eigenarten an Hand der den Astuarbewohnern zu Gebote stehenden Kompensationsmöglichkeiten hier näher zu betrachten. 
Über das Leben in Astuarien sind in den letzten Jahren zahlreiche Originalarbeiten und mehrere Reviews erschienen. Die meisten dieser Abhandlungen befassen sich vor allem mit verbreitungsökologischen oder physiographischen Aspekten. In unserem Zusammenhang sind folgende Arbeiten von besonderer Wichtigkeit: Caspers (1948, 1955, 1958, 1959), DAy (1951), SMith (1956), EMERy et al. (1957), Garms (1961), Kothe (1961), Kötter (1961), Reid (1961), Schulz (1961) und Schumacher (1961).

\section{SALINITAT UND TEMPERATUR IN ASTUARIEN}

Die Salinität der Ästuarien unterliegt häufig erheblichen Veränderungen. Im allgemeinen ist sie geringer als im Meerwasser. In Gegenden mit hohen Evaporationsraten und wenig Regen können bodennahe Salzgehaltswerte aber sogar 100\% schreiten. Auch die ionale Zusammensetzung und die relativen Anteile anderer gelöster Substanzen sind oftmals recht unterschiedlich. Im allgemeinen ist das Verhältnis von Karbonaten und Sulfaten zu Chloriden und das von Calcium zu Natrium höher als im Meerwasser (Clarke 1924). Ortliche Modifikationen werden vielfach verursacht durch (1) die Bodenart des Flußbettes und unmittelbar benachbarter Areale, (2) die Floren und Faunen, mit denen das Flußwasser in Berührung kommt, und (3) industrielle oder zivilisatorische Abfallprodukte, welche in den Fluß gelangen. Da das flußaufwärts sich vorschiebende Meerwasser und das seewärts ablaufende Flußwasser der Tendenz unterliegen, jeweils nach ihrer rechten Seite hin abgedrängt zu werden, ist die Salinität der linken Seite größerer Ästuarien auf der nördlichen Hemisphäre gewöhnlich höher. So hat PrITCHARd (1952) zum Beispiel festgestellt, daß die Salinität am linken Ufer des James-Flusses im Durchschnitt um 1\% höher ist als am rechten Ufer. Derartige Unterschiede können Verbreitung, Abundanz und physiologische Reaktionen ästuariner Organismen beeinflussen.

Die Temperatur des Ästuarwassers zeigt im allgemeinen stärkere jährliche und tägliche Fluktuationen als die des benachbarten Meeres, insbesondere wenn das Ästuar flach ist und das Wasser - etwa bei einkommender Flut - mit zuvor luftexponierten Bodenbereichen in Berührung kommt. Im Mündungsbereich des Ästuariums ist die Wassertemperatur etwa die gleiche wie im künstlichen Meerbereich; stromaufwärts nehmen die Unterschiede jedoch gewöhnlich schnell zu (siehe beispielsweise Sumner et al. 1914). Zusätzlich zu derartigen horizontalen Gradienten existieren vielfach starke vertikale Gradienten, besonders wenn warmes Flußwasser über kaltem Bradk- oder Meerwasser höherer Dichte abläuft.

\section{KOMPENSATIONSMOGLICHKEITEN}

Asstuarine Organismen verfügen über eine Reihe von Eigenschaften, welche ihnen gestatten, negative Wirkungen unerwünschter Salinitäts- oder Temperaturintensitäten bis zu einem gewissen Grade zu kompensieren. Einige der Studien, welche sich mit derartigen Eigenschaften befaßt haben, sollen hier besprochen werden, und zwar unter vier Aspekten: Ausweichreaktionen, Kontaktverminderungen, Regulatio- 
nen und Adaptationen. Die physiologisch sehr interessante Tatsache, daß ästuarine Organismen ganz generell eine erhöhte Resistenz gegenüber extremen Faktorintensitäten aufweisen, wird nicht näher erörtert. Eine derartige erhöbte Widerstandsfähigkeit ist in mehreren Fällen sowohl am isolierten Gewebe als auch am intakten Ganztier nachgewiesen worden. Sie ist ökologisch von größter Bedeutung und stellt offenbar eine wichtige Voraussetzung dar für die erfolgreiche Besiedlung ästuarer Lebensbezirke.

\section{Ausweichreaktionen}

Direktes Ausweichen im Sinne eines Ortswechsels - einer Abwanderung in zusagendere Salinitäts- oder Temperaturbedingungen - ist eine Kompensationsmöglichkeit, die nur vagilen Individuen zu Gebote steht und die sich naturgemäß auf Situationen beschränkt, in denen günstige Salzgehalts- oder Temperaturverhältnisse tatsächlich vorhanden und erreichbar sind. Vielfach führen vertikale und horizontale Ausweichbewegungen relativ rasch zu andersartigen Salinitäts- oder Temperaturintensitäten. Sie spielen daher als Simultankompensationen offenbar eine wichtige Rolle.

Im Substrat des Astuarbodens sind Salzgehalts- und Temperaturfluktuationen häufig wesentlich weniger ausgeprägt als im freien Wasser unmittelbar darüber. Auch die entsprechenden Mittelwerte können an beiden Orten recht unterschiedlich sein. Das ist in besonderem Maße der Fall in jenem Astuarbereich, in dem die prozentualen Anteile von Meer- und Flußwasser in Rhythmus der Gezeiten starken Schwankungen unterliegen (Abb. 1). Für viele Organismen stellt das Substrat daher ein potentielles

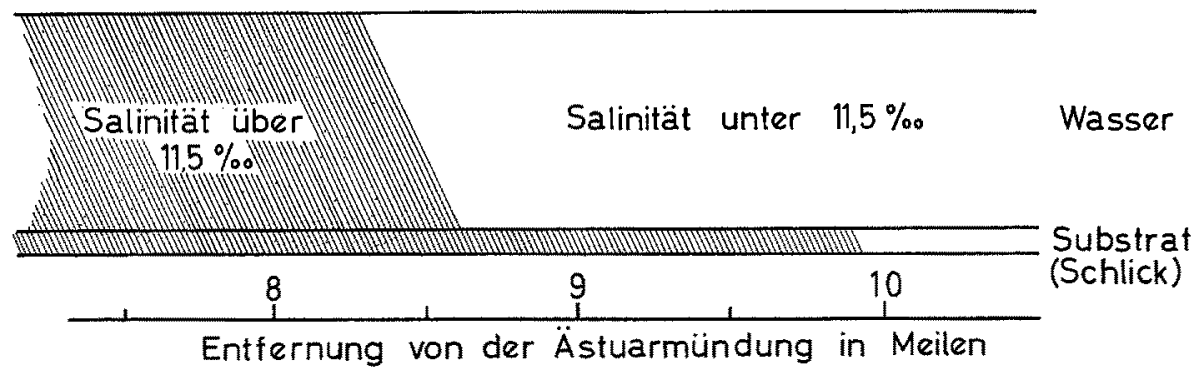

Abb. 1: Diagrammatische Illustration der Salinitätsverhältnisse in einem Astuar während des Niedrigwassers. Mit zunehmender Entfernung von der Ästuarmündung nimmt der Salzgehalt im freien. Wasser schneller ab (und ist größeren Fluktuationen unterworfen) als im Substrat.

(Nach AleXANDer et al. 1935; modifiziert)

Refugium dar. Permanente Substratbewohner genießen ganz allgemein stabilere und weniger extreme Salinitäts- und Temperaturbedingungen als ihre pelagischen Mitbewohner und unterscheiden sich von diesen daher häufig in ihrem Verbreitungsmuster. Derartige Unterschiede lassen sich auch bei Arten nachweisen mit ganz ähnlichen Resistenzgrenzen und Reaktionsamplituden. Dieser Umstand hatte früher zu verschiedenen Spekulationen Anlaß gegeben. Eine plausible Erklärung für die unterschiedliche Verbreitung wurde erst möglich, nachdem der Nachweis der andersartigen Salzgehaltsund Temperaturverhältnisse im und über dem Boden erbracht worden war. 
Umweltfaktoren können einander in ihrer Wirkung auf den lebenden Organismus beeinträchtigen. So kann die Wirkung eines Faktors A beispielsweise durch die Faktoren $\mathrm{B}$ und $\mathrm{C}$ verstärkt, geschwächt oder gar qualitativ modifiziert werden. Faktor $\mathrm{A}$ kann ferner die kritische untere und obere Grenzintensität des Faktors B verändern, also den zuträglichen Intensitätsbereich erweitern, verengen oder verschieben. Für Temperatur und Salzgehalt sind derartige Wirkungsbeziehungen von KINNE mehrfach nachgewiesen und 1956 (a, b) eingehender erörtert worden (siehe auch JørGENSEN 1960, McCombie 1960, Lewin \& Gulllard 1963, Kinne 1964b). Neben dem oben erwähnten direkten Ausweichen der negativen Wirkung eines bestimmten Umweltfaktors, welche die Erreichbarkeit günstigerer Faktorintensitäten zur Voraussetzung hat, steht ästuarinen Organismen daher vermutlich auch die Möglichkeit eines "indirekten A asweichens" offen. Ein Beispiel möge das erläutern: Wenn die Salinität eine kritische Intensität erreicht und günstigere Salzgehaltsverhältnisse zur Zeit nicht erreichbar sind, könnten die negativen Salinitätswirkungen durch Aufsuchen geeigneter Temperatur- oder Lichtbedingungen geschwächt und somit - wenigstens zum Teil - kompensiert werden. Ein eindeutig analysierter Fall eines derartigen „indirekten Ausweichens" unter natürlichen Bedingungen im Ästuar ist mir nicht bekannt. Die ständig wachsende Anzahl experimenteller Ergebnisse über die Wirkungsbeziehungen zwischen Temperatur, Salzgehalt und anderen Faktoren macht das Vorhandensein einer indirekten Ausweidreaktion jedoch sehr wahrscheinlich.

\section{Kontaktverminderungen}

Eine Verminderung des unmittelbaren Kontaktes mit ungünstigen oder gar schädlichen Faktorintensitäten des Außenmediums ist eine zeitlich vielfach recht eng begrenzte Kompensationsmöglichkeit. Kontaktverminderungen treten im allgemeinen im Gefolge von starken plötzlichen Salinitäts- oder Temperaturveränderungen auf. Sie können aber auch durch langanhaltende, extreme Stresslagen ausgelöst werden, etwa durch lange Perioden geringen Salzgehaltes oder niedriger Wintertemperaturen.

Temporäre Extremsalinitäten können in wesentlichen 7 verschiedene Typen der Kontaktverminderung verursachen: (1) Absonderung von schleim- oder gallertartigen Substanzen, welche die empfindlichen Körperoberflächen schützen; derartige Reaktionen sind bei einer ganzen Reihe von Organismen beobachtet worden, insbesondere bei Anneliden, Mollusken und Fischen. (2) Rückzug in Wohnhöhlen, Röhren oder Felsspalten, deren Eingänge mitunter noch besonders verschlossen werden können (manche Polychaeten und Krebse). (3) Muskelkontraktionen, welche zu einer Verringerung des Oberflächen/Volumen-Quotienten empfindlicher Körperpartien oder des Gesamtkörpers führen (Hydroidpolypen, wurmartige Formen, Gastropoden). (4) Einziehen empfindlicher Organe wie etwa der Tentakel bei Cnidariern oder des gesamten Weichkörpers bei gehäusebauenden Protozoen, Bryozoen etc. (5) Schalenverschluß; manche Lamellibranchiaten- und Balanidenarten können beispielsweise an sich letal wirkende, extrem niedrige oder hohe Durchschnittssalinitäten permanent ertragen, wenn die $z$ wischenzeitlichen Klimaverbesserungen (Salzgehaltsfluktuationen) ihnen genügend Zeit zum Fressen und zur Abgabe von Stoffwechselendprodukten belassen. 
(6) Veränderungen der Körperform und anderer struktureller Eigenarten können auftreten nach länger anhaltenden Extremlagen; Beispiele: Veränderungen von Koloniem wuchs, Hydrantengröße und Tentakelzahl bei den Hydroidpolypen Cordylophora caspia, Perigonimus megas und Laomedea loveni; weitere Beispiele liefern Protozoen, Schwämme und einige Crustaceen. (7) Transformation in Ruhestadien, welche beispielsweise bei Bakterien, Pilzen, Protozoen, Schwämmen, Hydrozoen, Bryozoen, Rotatorien, Nematoden, Tardigraden und Crustaceen vorkommen. Bei diesen Formen führen Sporen-, Cysten- oder Menontenbildungen vielfach zu einer erheblichen Verminderung des unmittelbaren Kontaktes mit dem umgebenden Medium. Die Transformation in Ruhestadien geht einher mit einer Reduktion der Aktivität und Stoffwechselintensität und resultiert im allgemeinen in einer starken Erhöhung der Umweltresistenz. Auf diese Weise können längere, im Zustand aktiven Lebens nicht $\mathrm{zu}$ ertragende Klimaverschlechterungen überdauert werden. Nach Beendigung der Klimaverschlechterung tritt dann eine Rückwandlung in die aktive Kondition ein.

Temporäre Extremtemperaturen sind weitaus gefährlicher in ihren Auswirkungen. Obgleich sie in manchen Fällen ähnliche Reaktionen auslösen wie Extremsalinitäten, kommt eigentlich nur der Transformation in Ruhestadien - vor allem während des Winters - größere Bedeutung zu. Ansonsten sind die ästuarinen Organismen kurzfristigen, plötzlich auftretenden Temperaturextremen recht hilflos ausgeliefert.

\section{Regulationen}

Die meisten ästuarinen Organismen verfügen über beachtliche Möglichkeiten, die physiko-chemischen Reaktionsbedingungen ihres Körperinneren gegenüber Veränderungen im umgebenden Medium zu regulieren. Ihre Regulationsfähigkeiten beschränken sich allerdings nahezu vollkommen auf den Salzgehaltsfaktor; hinsichtlich der Temperatur sind sie im wesentlichen wechselwarme Konformisten, denen wirkungsvolle Mechanismen zur Temperaturregulation fehlen. Temperaturfluktuationen beeinflussen ihre Lebensprozesse daher direkt und in ihrer ganzen Härte, soweit ihre schädigenden Wirkungen nicht durch Akklimatisationen (p. 148) oder durch Transformationen in Ruhestadien (s. o.) etwas abgefangen werden. Wir beschäftigen uns aus diesem Grunde im vorliegenden Kapitel ausschließlich mit dem Salinitätsfaktor.

Zur Kompensation ungünstiger Salinitäten stehen drei Regulationsprinzipien zur Verfügung: Ionenregulation, Volumregulation und Osmoregulation. Neuere Reviews über diese Regulationsprinzipien sind veröffentlicht worden von Brown \& Dantelli (1955), Beadle (1957), Robertson (1957, 1960a, b), Remane \& Schlieper (1958), Prosser \& Brown (1961), Lockwood (1962) und Kinne (1963b, $1964 a, b, c)$.

\section{Ionenregulation}

Die Fähigkeit zur Ionenregulation ist phylogenetisch offenbar sehr alt - älter als die zur Volum- oder Osmoregulation. Alle bisher untersuchten aquatischen Organismen sind in der Lage, die ionale Zusammensetzung ihrer Körper-, Gewebe- oder Zellflïssigkeiten in gewissem Ausmaß zu regulieren. 
Im Pflanzen reich ist die Fähigkeit zur Ionenregulation bei einer ganzen Reihe aquatischer Vertreter nachgewiesen oder doch sehr wahrscheinlich gemacht worden: Blinks (1951), Scott \& Hayward (1953, 1954, 1955), Biebl (1956), Bergquist (1958a, b), Eprley (1958a, b, 1962), MacRobbie \& Dainty (1958a, b), Eppley \& Cyrus (1960), Hope \& Walker (1960) und Gullard (1962). Allerdings haben wir nur wenige Informationen zur Hand über Ionenregulation bei Algen, welche sich unter osmotischem Stress befinden. Bei Porpbyra perforata ist die Uberlebensfähigkeit in verdünntem und überkonzentriertem Seewasser von der Menge des vorhandenen $\mathrm{Ca}$ abhängig. Ca scheint besonders hinsichtlich der Erhaltung der Membranselektivität für $\mathrm{K}$ und $\mathrm{Na}$ von Bedeutung zu sein. Über einen weiten Salinitätsbereich (etwa 3,5 bis $70 \%$ ) vermögen die Zellen der P. perforata $\mathrm{K}$ anzureichern und die Na-Konzentrationen niedrigzuhalten. Starke Aussüßung durch Regengüsse führt jedoch zu einem Zusammenbruch des Ionentransportmechanismus und stellt daher eine größere Existenzbedrohung dar als erhöhte Salinitäten. Während der Ebbe vermag ein starker Regenguß den normalerweise auf der Algenoberfä̈he befindlichen Meerwasserfilm praktisch vollkommen abzuwaschen. Das kann dann den Verlust der Membranselektivität sowie eine Reduktion der Atmungsintensität und schließlich tödlichen Ionenverlust zur Folge haben (EPPLEY \& CYRus 1960).

Ahnlich liegen die Dinge bei Chaetomorpha linum, einer grünen Gezeitenalge der deutschen Nordseeküste. C. linum kompensiert Veränderungen im osmotischen Potential des Außenmediums durch Turgorregulationen, welche vor allem durch aktive Anreicherung oder Abstoßung von $\mathrm{K}$ und $\mathrm{Cl}$ bewerkstelligt werden (Kesseler 1959). Die Anreicherung von $\mathrm{KCl}$ gegen einen starken Diffusionsgradienten beansprucht Energie, die nach Kesseler (1962) durch die Atmung freigesetzt wird. Das quantitativ dominierende Kation des Meerwassers, nämlich das $\mathrm{Na}$, ist für die positive und negative Turgorregulation kaum von Bedeutung. Ca-Mangel im Außenmedium dagegen führt zu einer erhöhten Abgabe von $\mathrm{KCl}$ und beträchtlichem Turgorverlust (Kesseler 1964). Nach einer mündlichen Mitteilung von Herrn Dr. Kesseler ist $\mathrm{K}$ in den meisten der bisher untersuchten Algen aus den Regionen von Helgoland und Neapel das dominierende intrazelluläre Kation; höhere Na-Konzentrationen als im Meerwasser wurden nur bei einigen Siphonales festgestellt.

Ein Sonderfall ist übrigens von einigen Arten der Gattung Desmarestia bekannt geworden: sie speichern Schwefelsäure in ihren Vakuolen, wobei die $\mathrm{SO}_{4}$-Ionen mengenmäßig das Hauptanion stellen und die Elektroneutralität durch H-Ionen zustande gebracht wird (EPPLEY \& BOVELL 1958). Bei einer Reihe von Algen spielt Na eine Rolle im Zusammenhang mit den "Ionenpumpen“ (ERPLEY \& Cyrus 1960, Hope \& Walker 1960, EPPLey 1962, Lewin 1962).

Die Bedeutung der ionalen Zusammensetzung des umgebenden Wassers für das Wachstum und die Vermehrung aquatischer Pflanzen wird durch eine ganze Reihe von Arbeiten belegt. Ich verweise hier besonders auf die zusammenfassenden Darstellungen von Provasoli (1958), Guillard (1962) und Lewin \& Guillard (1963) sowie auf die Originalarbeiten von VollenweIder (1950), Provasoli et al. (1954), Droop (1958), Schmitz (1959), Guillard \& Ryther (1962) und Williams (1962). Provasoli et al. (1954) haben sich insbesondere mit der Frage beschäftigt, inwieweit die Mineralbedürfnisse mariner und limnischer Phytoflagellaten die ionale Komposition ihrer natürlichen 
Habitatgewässer widerspiegeln. In ihren Experimenten variierten sie die Salinität sowie folgende Ionen-Relationen: Monovalente/Divalente (M/D) und $\mathrm{Ca} / \mathrm{Mg}$, und zwar mit dem Ziel, die relative oder absolute Bedeutung dieser Faktoren für das Wachstum zu ermitteln. Es ergab sich eine zum Teil erhebliche Plastizität der Versuchsobjekte. Für jede der getesteten Flagellatenarten konnte festgestellt werden, daß $\mathrm{Ca}$ und $\mathrm{Mg}$ bei einer bestimmten Salinität und $\mathrm{M} / \mathrm{D}$-Relation in größerem Ausmaße gegeneinander austauschbar sind, und daß auch Deviationen von dieser Versuchsanordnung noch maximale Wachstumsraten gestatten. Bei Salinitäten und M/D-Relationen, die in etwa den ursprünglichen Habitatbedingungen der Versuchsobjekte glichen, bevorzugten die marinen Vertreter erwartungsgemäß $\mathrm{Ca} / \mathrm{Mg}$-Relationen von etwa 1:3 (wie sie im Meerwasser realisiert sind), während die limnischen Vertreter $\mathrm{Ca} / \mathrm{Mg}$-Relationen von etwa $6: 1$ bevorzugten (wie sie im Süßwasser vorkommen).

Auch Droop (1958) stellte bei seinen Untersuchungsobjekten eine erstaunliche Toleranz gegenüber Veränderungen in der ionalen Zusammensetzung des Außenmediums fest; nahezu über den gesamten Bereich von Meerwasser bis Süßwasser ertrugen sie die gebotenen Veränderungen in der $\mathrm{Ca} / \mathrm{Mg}$-Relation. Die sehr eurysalinen Formen Monochrysis lutheri, Hemiselmis virescens und Nannochloris oculata wuchsen am besten bei $\mathrm{Ca} / \mathrm{Mg}$-Relationen, welche den Verhältnissen im Meer ähnelten, während die weniger eurysaline Meeresdiatomee Skeletonema costatum überraschenderweise abweichendere Relationen bevorzugte. $S$. costatum unterscheidet sich jedoch von den genannten eurysalinen Arten dadurch, daß sie eine engere Na-Amplitude besitzt. Droop weist in seiner Arbeit auf Informationen von BraArud (1951) und Braarud \& Pappas (1951) hin, nach denen die untere limitierende Na-Grenze bei marin-neritischen Dinoflagellaten bei etwa $3000 \mathrm{mg} / 1$ liegt, während sie sich bei supra-litoralen Arten bis zu etwa $300 \mathrm{mg} / \mathrm{l}$ erniedrigen kann. Die ozeanische Rhodomonas lens hat nach Provasoli et al. (1954) offenbar eine untere Na-Grenze von nicht weniger als $4000 \mathrm{mg} / 1$. Nach diesen Untersuchungsergebnissen scheint die Na-Toleranz der Meerespflanzen ein wichtiger limitierender Faktor zu sein bei der Besiedlung von Aistuarien und anderen Gebieten mit herabgesetzter Salinität oder modifizierter ionaler Komposition. Bei Thalassiosira fuviatilis hängt die Salinität, bei welcher maximales Wachstum erfolgt, von der $\mathrm{SO}_{4} /$ Cl-Relation ab. Derartige Verschiebungen der zuträglichen Gesamtkonzentration könnten bei der Besiedlung von Gebieten mit reduzierter Salinität ebenfalls von Bedeutung sein.

Insgesamt geht aus den uns zur Zeit aus dem Pflanzenreich vorliegenden Informationen hervor, daß kleinere Veränderungen in den Tonenrelationen recht gut vertragen werden und daher von geringer physio-ökologischer Bedeutung sind, daß signifikante Modifikationen aber Letalgrenzen und Salinitätspräferenzen ebenso beeinflussen können wie Wachstum und Vermehrung und somit auch Konkurrenzvermögen und Verbreitung der betroffenen Formen.

Im Tier reich ist die Fähigkeit zur Ionenregulation in einer Fülle von Beiträgen behandelt worden (jüngste Reviews: KINNE 1963b, 1964a, b). Die meisten salzwasserlebenden Tiere zeigen eine Tendenz, den Gehalt an K- und H-Ionen in ihren Körperflüssigkeiten und Geweben - relativ zum Außenmedium - zu erhöhen, den Gehalt an $\mathrm{Na}$ - und $\mathrm{Cl}$-Ionen dagegen zu erniedrigen und sich den im Seewasser häufigen $\mathrm{Mg}$ - und $\mathrm{SO}_{4}$-Ionen weitgehend $\mathrm{zu}$ verschließen. In ein verdünntes Außenmedium überführt, 
sind ästuarine Tiere im allgemeinen in der Lage, die ursprünglichen Ionenregulationen in ihrem Körperinneren weitgehend aufrechtzuerhalten. Bei ihren marinen Verwandten dagegen ist die Fähigkeit zur Ionenregulation wesentlich geringer ausgeprägt. So stimmt die ionale Zusammensetzung der Körperflüssigkeiten bei der im wesentlichen rein marinen Gruppe der Echinodermata sowohl im Meerwasser als auch im Brackwasser weitgehend mit derjenigen des Außenmediums überein. Als Beispiel sei hier der Seestern Asterias rubens erwähnt (Tab. 1). Die intrazellulären Ionenrelationen (Blut,

\section{Tabelle 1}

Ionale Zusammensetzung der Körperflüssigkeit bei Asterias rubens nach Aufenthalt in Meerwasser von $31 \%$ Salzgehalt (nach CoLe 1940) beziehungsweise in Brackwasser von etwa $16 \%$ Salzgehalt (nach REMANE \& SCHLIEPER 1958 und SECK 1958). Die Darstellung beschränkt sich auf die wichtigsten Ionen. Alle Konzentrationen sind in $\mathrm{mM} / \mathrm{l}$ angegeben

\begin{tabular}{|lllllll|}
\hline Medium & $\mathrm{Na}^{+}$ & $\mathrm{K}^{+}$ & $\mathrm{Ca}^{++}$ & $\mathrm{Mg}^{++}$ & $\mathrm{Cl}-$ & $\mathrm{SO}_{4}-$ \\
\hline $31 \% 0 \mathrm{~S}$ & 417 & 9,10 & 9,41 & 50,1 & 483 & 30,3 \\
Körperflüssigkeit & 412 & 9,56 & 9,31 & 50,0 & 488 & 30,1 \\
16\%0 S & 215 & 4,98 & 5,56 & 24,05 & 252,8 & 13,08 \\
Körperflüssigkeit & 216 & 5,40 & 5,60 & 24,22 & 255 & 13,05 \\
\hline
\end{tabular}

Muskeln, Körperwand) weisen allerdings art- oder gruppenspezifische Unterschiede auf, ebenso die Echinodermeneier (Rothschild \& Barnes 1953). Cnidaria, Annelida und Mollusca sind bessere Ionenregulierer. Die meisten ästuarinen Krebse verfügen über bemerkenswerte ionenregulatorische Fähigkeiten (RoBERTsON 1957, 1960a, b). Gleiches gilt für die Fische; die bisher untersuchten ästuarlebenden Knochenfische erwiesen sich fast ausnahmslos als bessere Ionenregulierer als ihre wirbellosen Mitbewohner.

Ionenregulationen erfolgen bei wasserlebenden Tieren zunächst zwischen Außenmedium und Blut. Etwaige im Blut auftretende Schwankungen können dann nochmals beim Übergang Blut/Zelle verringert werden, so daß die intrazellulären Modifikationen - wie bereits am Beispiel der Echinodermen angedeutet - vielfach in recht engen Grenzen bleiben. Die meisten der daraufhin geprïtten ästuarinen Tiere zeigen signifikante Unterschiede in der ionalen Zusammensetzung von Körper- und Zellfiüssigkeiten. Zwar ist die Gesamtosmokonzentration der Zellflüssigkeit mit derjenigen von Blut und

Tabelle 2

Chloridmengen im Blut und Muskelgewebe (Fuß) der Miesmuschel Mytilus edulis (1) in normalem Meerwasser, (2) 24 Stunden und (3) 48 Stunden nach Überführung in verdünntes Meerwasser. Alle Konzentrationen sind in $\mathrm{mM} / \mathrm{kg} \mathrm{H} \mathrm{H}_{2} \mathrm{O}$ angegeben. (Nach Kroge 1938)

\begin{tabular}{|lccc|}
\hline Versuchsbedingung & Außenmedium-Cl- & Blut-Cl- & Muskel-Cl- \\
\hline (1) Meerwasser & 532 & 526 & 189 \\
(2) Verdünntes Meerwasser & 284 & 283 & 100 \\
nach 24 Stunden & 229 & 224 & 55 \\
\hline
\end{tabular}


Coelomflüssigkeit im allgemeinen weitgehend isosmotisch; außer aus anorganischen Ionen besteht sie jedoch aus größeren Mengen organischer Substanzen, während die Gesamtosmokonzentration von Blut und Coelomflüssigkeit größtenteils nur durch anorganische Ionen zustande kommt, vor allem durch $\mathrm{Na}$ und $\mathrm{Cl}$. Bei Gammarus duebeni zum Beispiel macht das Gewebe-Chlorid nur einen Bruchteil des Blut-Chlorids aus tiber einen Salzgehaltsbereich von Meerwasser bis Süßwasser (BeAdLe \& CRAGg 1940a, b), und bei Mytilus edulis beträgt das Gewebe-Chlorid (Fußgewebe) in $35 \%$ und Brackwasser weniger als die Hälfte des Blut-Chlorids (Tab. 2).

Die molekularen Mechanismen des aktiven Ionentransportes sind noch nicht in ausreichendem Maße erforscht (siehe z. B. Kinne 1963b, 1964b). Ackerman (1962) hat jedoch nachgewiesen, daß aktiver Transport letztlich metabolische Energie beansprucht. Im Hinblick auf die Ionenregulation repräsentieren nach unseren heutigen Kenntnissen folgende physiologische Eigenschaften wichtige Voraussetzungen für eine erfolgreiche Besiedlung ästuariner Lebensräume: (1) Gewebetoleranz gegenüber Modifikationen in der ionalen Zusammensetzung des umgebenden Wassers, (2) hohe Fähigkeiten zur Ionenregulation und (3) osmotische Stabilisation des Körperinneren durch gelöste organische Substanzen.

\section{Volumregulation}

Offenbar sind die meisten Astuarbewohner in der Lage, ihr Körpervolumen zu regulieren. Im Gefolge einer plötzlichen Salinitätsveränderung gewinnen oder verlieren sie nicht nur Wasser, sondern auch Salze. Volumveränderungen deuten auf Verschiebungen im quasi-stationären Gleichgewicht des kontinuierlichen Austausches von Wasser und Salzen zwischen Organismus und Umwelt hin. Derartige Verschiebungen rufen bei Volumregulierern eine ganze Serie von Reaktionen und Kompensationen hervor, welche zu Veränderungen in der Permeabilität und den Exkretionsprozessen führen und schließlich einen neuen stationären Zustand herstellen. Im allgemeinen dauern Volumkompensationen Minuten oder Stunden; sie können sich jedoch auch über Tage erstrecken.

Bei einer Reihe von eurysalinen Protozoen wird die Volumregulation vermutlich primär durch die kontraktile Vakuole bewirkt (siehe z. B. MüLLER 1936, OBERTHÜR 1937, KrTcurng 1938, 1948, 1951, 1952, 1954). Es gibt jedoch einige Protozoenarten, denen eine kontraktile Vakuole fehlt, die aber nichtsdestoweniger in der Lage sind, ihr Körpervolumen aktiv zu regulieren. Die Rotalge Porphyra perforata vermag ihr osmotisches Volumen über einen Salinitätsbereich von 17 bis $35 \%$ nahezu konstant zu halten. Eppley \& Cyrus (1960) vermuten, daß dieses Regulationsvermögen vor allem auf differentielles Schrumpfen und Schwellen der strukturellen Polysaccharide zurückzuführen ist, aber nicht auf aktive Wassersekretion. Weitere Beispiele von Volumregulierern sind Nereis diversicolor (SchlIEPER 1929a, b, BeAdLE 1931, 1937, Ellis 1937, 1939), Nereis virens (Topring \& FULLER 1942), Marphysia gravelyi (KRISHNAMOORTHI 1951; siehe auch Abb. 2), Pbyscosoma japonicum (Koller 1939), Procerodes ulvae (Beadle 1934, Weil \& Pantin 1931, Pantin 1931b; siehe auch Abb. 3), Carcinus maenas (NAGeL 1934, Bethe et al. 1935), Gasterosteus leiurus (Gueylard 1925), Pleuronectes pla- 


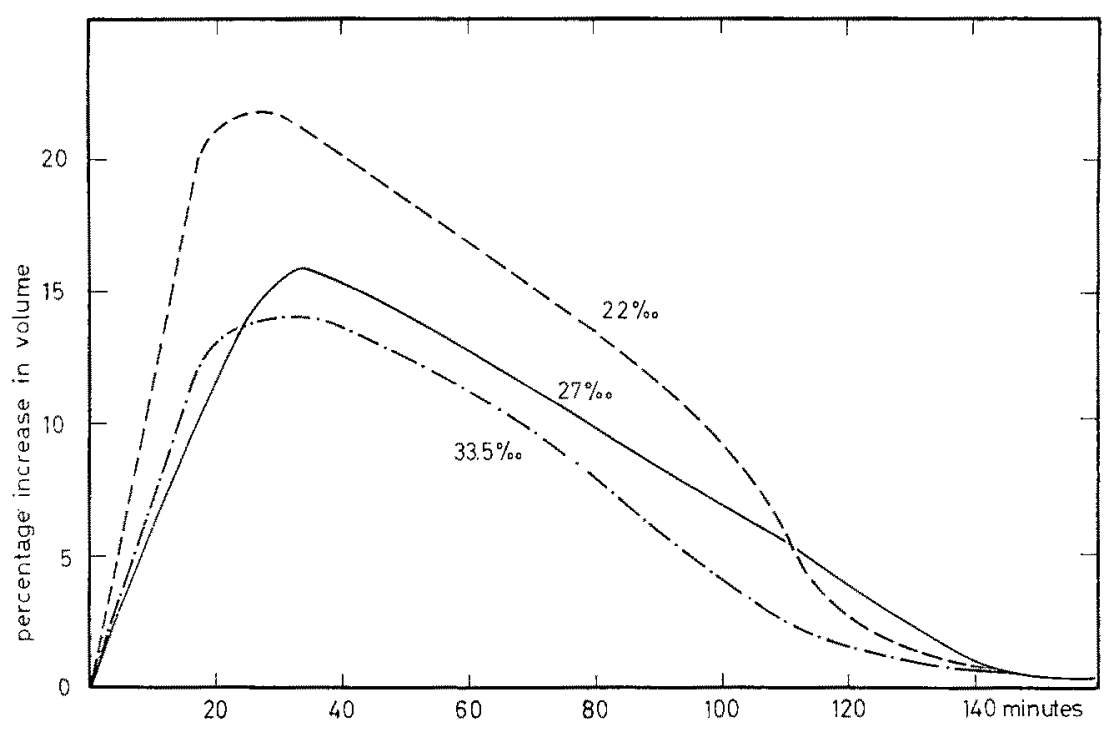

Abb. 2: Volumregulation bei Larven des Polychaeten Marphysia gravelyi. Die ausgeglichenen Kurvenzüge geben die prozentualen Volumveränderungen in Wasser reduzierter Salinität wieder. (Nach KrishNAMOoRTHi 1951; modifiziert)

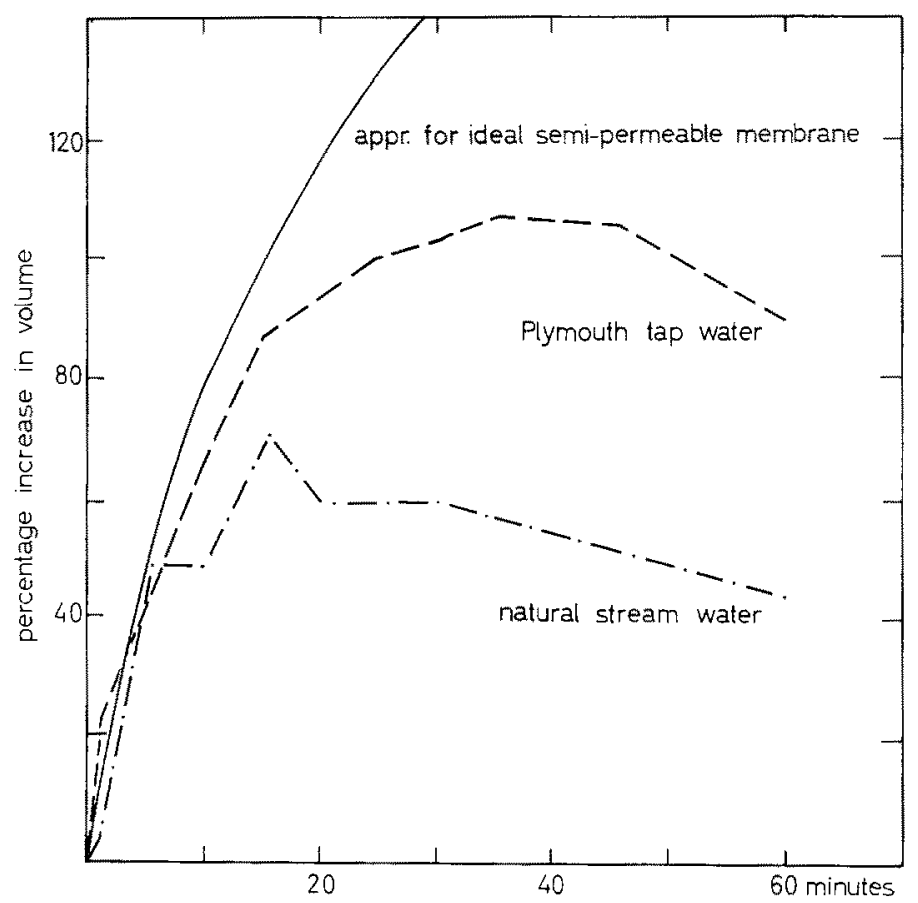

Abb. 3: Volumregulation bei dem Turbellar Procerodes (Gunda) ulvae in Leitungswasser und natïrlichem Flußwasser. (Nach PANTrN 1931b; modifiziert) 
tessa (Henschel 1936). Zwei Beispiele seien etwas ausführlicher dargestellt. Das triclade Turbellar Procerodes (früher: Gunda) ulvae lebt in den Astuarien von Devonshire, welche durch erhebliche tägliche Salinitätsschwankungen ausgezeichnet sind.

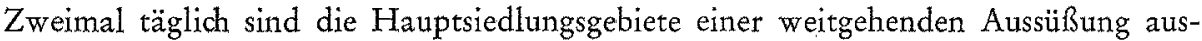
gesetzt. Wenn man diese Turbellarien im Experiment in destilliertes Wasser oder in weiches Leitungswasser überführt, so nimmt ihr Volumen rasch immer mehr zu und schließlich platzen sie. Die Volumregulation funktioniert nur in normalem, hartem Süßwasser: Nach Überführung in natürliches Flußwasser steigt das Körpervolumen um etwa $70 \%$ und beginnt sich dann wieder etwas zu verringern (Abb. 3). In dieser Kondition können die Tiere mehrere Tage überleben (Wenl \& Pantin 1931). Der Regulationsmechanismus von Procerodes beruht zum Teil auf Veränderungen in der Membranpermeabilität, welche ihrerseits vom Akklimatisationszustand abhängen und entscheidend durch den Ca-Gehalt des umgebenden Wassers beeinflußt werden, zum anderen Teil auf die Wasserentfernung durch das Darmepithel. Dieser letztere Aspekt wird durch Abbildung 4 verdeutlicht. Ein Teil des Wasserïberflusses wird nach PANTIN (1931a, b) während der Aussüßungsphase in den Darmzellen gesammelt. Es ist denk-
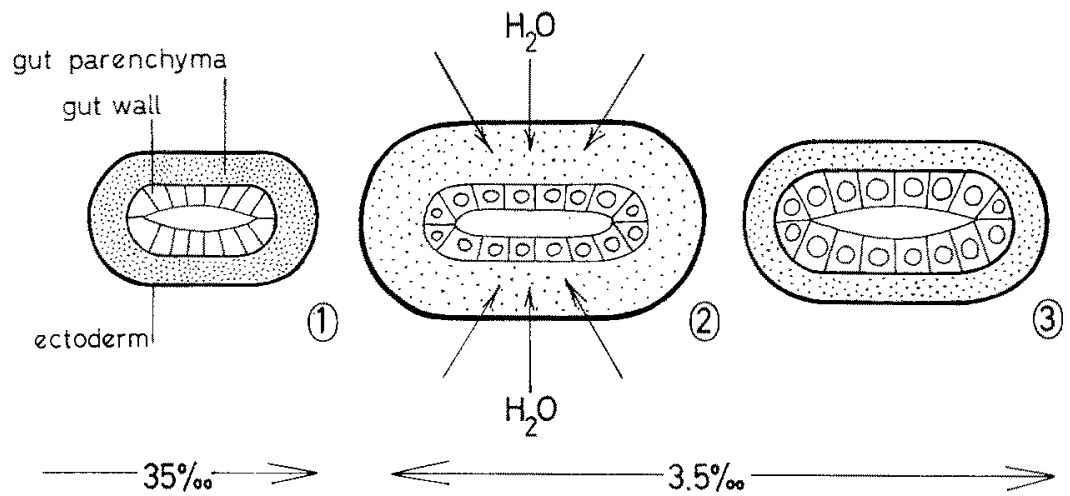

Abb. 4: Schematische Demonstration der Wasserentfernung durch das Darmepithel bei dem Turbellar Procerodes ulvae. Dargestellt sind Querschnitte (1) nach Aufenthalt in unverdünntem Meerwasser von etwa $35 \%$ S; (2) einige Stunden nach Uberführung in 3,5\%0: Wasser ist eingedrungen und hat erhebliche Schwellungen im Parenchym verursacht; die Darmepithelzellen beginnen in intrazellulären Vakuolen Wasser zu sammeln; (3) etwa 12 Stunden nach der Überführung; die Darmzellen haben den größten Teil des Wassers entfernt.

(Nach BEADLE 1934; modifiziert)

bar, daß die Darmzellen Wasser gegen den osmotischen Gradienten nach außen befördern können; auf jeden Fall wird das im Darmepithel gespeicherte Wasser während der Flut (Erhöhung der Salinität des umgebenden Wassers) wieder abgeführt. Auch der ästuarine Polychaet Nereis diversicolor bedient sich einer Kombination verschiedenartiger Regulationsmechanismen. Nach einer durch Salzgehaltserniedrigung bewirkten Volumzunahme setzt in den Nephridien eine erhöhte Urinproduktion ein (BEADLE 1937); gleichzeitig wird der durch die Salzgehaltserniedrigung bewirkte Salzverlust durch eine Verstärkung der aktiven Salzaufnahme kompensiert (FRETTER 1955, JøRGENSEN \& DALEs 1957). In sehr niedrigen Salinitäten ist $N$. diversicolor offenbar 
in der Lage, einen Teil der Salze in seinen Nephridien zu reabsorbieren und auf diese Weise hypoosmotischen Harn zu bereiten (siehe auch POTTs \& PARRY 1963).

In engem Zusammenhang mit den Volumveränderungen stehen die Veränderungen des Körpergewichts. Durch osmotische Wasseraufnahme bedingte Volumzunahme ist natürlich stets verbunden mit einer Gewichtszunahme. Aber Gewichtszunahme - etwa bei hartschaligen Formen - braucht nicht immer einherzugehen mit einer Zunahme des Körpervolumens. Ein Beispiel für salzgehaltsbedingte Gewichtsveränderungen ist in Abbildung 5 dargestellt. Bei dem süßwasserlebenden Gobio fluviatilis verursacht eine Uberführung in bluthyperosmotisches Brackwasser irreversiblen, letalwirkenden Wasser- und Gewichtsverlust. In einer ähnlichen Stress-Situation gelingt es dem euryplastischeren Gasterosteus leiurus dagegen, den anfänglichen Wasser- und Gewichtsverlust $\mathrm{zu}$ kompensieren und auf diese Weise einen plötzlichen Salinitätsanstieg zu überleben.

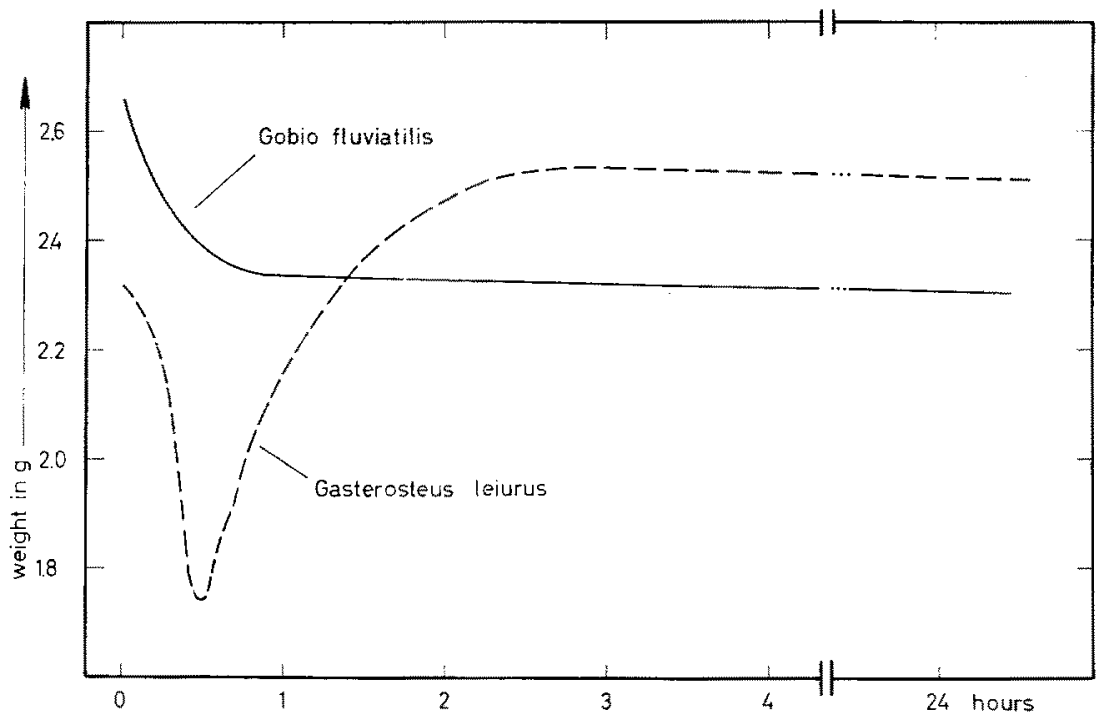

Abb. 5: Veränderungen im Körpergewicht bei dem stenosalinen Süß\}asserfisch Gobio fwviatilis nach Uberführung in Brackwasser von etwa $12 \%$ und bei dem eurysalinen Fisch Gasterosteus leiurus nach Uberführung in Wasser von etwa 37\%. (Nach GuEYLARD 1925; modifiziert)

\section{Osmoregulation}

Die Gesamtosmokonzentration der Zirkulations- und Gewebeflüsigkeiten ist, namentlich bei den komplexeren Lebensformen, von großer physiologischer Bedeutung. Im Gegensatz zu den Osmokonformisten der ozeanischen und rein marinen küstennahen Lebensbereiche sind Ästuarbewohner durchweg leistungsstarke Osmoregulierer. Sie vermögen die osmotische Konzentration des Körperinneren auch bei beträchtlichen Schwankungen der Salinität aufrechtzuerhalten.

Die meisten der bisher untersuchten aquatischen $\mathrm{Pfl}$ a $\mathrm{nz}$ en erhalten in ihrem Inneren eine Gesamtosmokonzentration, welche etwas höher liegt als die des um- 
gebenden Wassers. Ihre Fähigkeiten zum Ertragen extremer Salinitäten hängen im wesentlichen ab von ihrer protoplasmatischen Resistenz (GuilLaRd 1962). Im einzelnen gibt es verschiedene Regulationstypen mit recht unterschiedlichen Leistungen. Unter den marinen Algen zum Beispiel sind einige kaum hyperosmotisch, während andere in ihrem Inneren nahezu die doppelte Osmokonzentration aufweisen wie das umgebende Meerwasser. Brackwasseralgen sind dem Außenmedium gegenüber im allgemeinen stark hyperosmotisch. Süßwasserformen erzeugen vielfach sehr hohe osmotische Werte von etwa $5 \mathrm{~atm}$ (Burnks 1951). In enger Beziehung zur Osmokonzentration des Innenmediums steht der Turgor, der reelle hydrostatische Innendruck der Pflanzenzelle. Bei gleichbleibender Osmokonzentration des Innenmediums haben Salinitätsveränderungen eine Tendenz zur Turgorveränderung zur Folge: Salinitätserhöhung wirkt im Sinne einer Verringerung, Salinitätserniedrigung dagegen im Sinne einer Erhöhung des Turgors. Besonders starke Schwankungen der Osmokonzentration des umgebenden Wassers können daher zu einer gefährlichen Plasmolyse oder zum Platzen der Zellen führen (Höfler 1931, 1932, Brebl 1937, Collander 1939). Viele ästuarine Arten entgehen derartigen Gefahren durch eine Regulation ihres Turgors: bei Salinitätsveränderungen modifizieren sie die Osmokonzentration ihres Zellsaftes gleichsinnig und im gleichen Ausmaß; der Zellturgor, welcher ja die Differenz zwischen den Osmokonzentrationen von Außen- und Innenmedium darstellt, erreicht dann nach Beendigung der Regulation in etwa wieder seinen Ausgangswert. Das Vermögen, den Turgor zu regulieren, kann bei litoralen und ästuarinen Arten erheblich sein. Nach KESSELER (1959) vermag Cbaetomorpha linum über einen Salzgehaltsbereich von Süßwasser bis $35 \%$ einen relativ konstanten Turgor, nämlich 14,8 bis $16,5 \mathrm{~atm}$, aufrechtzuerhalten. Selbst bei extrem hohen Salzkonzentrationen weisen manche Arten erstaunliche Leistungen auf. So fand BüNNING (1934) noch bei beginnender Salzkristallisation turgeszente Elacbista vor. Die Mechanismen der Osmo- und Turgorregulation sowie andere Aspekte der Physiologie und Biochemie der Algen sind zusammenfassend dargestellt worden von BuInks (1951) und LEwIN (1962). Insbesondere mit den Mechanismen der Turgorregulation haben sich befaßt Drevs (1896), BuchHerm (1915) und Kesseler $(1960,1962,1964)$.

Unter den aquatischen W irbellosen und den Fischen vermögen die leistungsstärksten Osmoregulierer eine bemerkenswerte osmotische Stabilität ihres Innenmediums herbeizuführen. Viele der daraufhin untersuchten ästuarinen Vertreter können die Osmokonzentration ihrer Zirkulationsflüssigkeit über Salinitätsamplituden von 20 oder gar 30\% aufrechterhalren. Sie haben die physiko-chemischen Reaktionsbedingungen in ihrem Körperinneren damit weitgehend von den Salinitätsschwankungen im ästuarinen Lebensbereich unabhängig gemacht. Allerdings ist ein derartig ausgeprägtes Regulationspotential nicht in jedem Falle eine unabdingbare Voraussetzung für eine erfolgreiche Besiedlung von Astuarien: Eurysalinität kann auch auf einer großen Gewebetoleranz gegenüber Salinitätsfluktuationen beruhen oder auf besonders geringer Oberflächenpermeabilität.

Bezüglich ihrer osmoregulatorischen Leistungsfähigkeit lassen sich die tierischen Osmoregulierer in drei große Gruppen unterteilen, nämlich in eurysaline, holeurysaline und oligosaline Regulierer. Eurysaline Osmoregulierer (Abb. 6) können in ausreichendem Maße regulieren in Wasser mit reduzierter, erhöhter oder stark fluk- 
tuierender Salinität; über längere Zeiträume benötigen sie jedoch mehr Salz als es in reinem Süßwasser zur Verfügung steht. Beispiele sind die Polychaeten Nereis diversicolor (SCHLIEPER 1929a, b, 1930, SMITH 1955a, b, c, 1956), Nereis limnicola (früher lighti) (Smrth 1957, 1959); die Crustaceen Gammarus locusta, G. obtusatus (Beadle

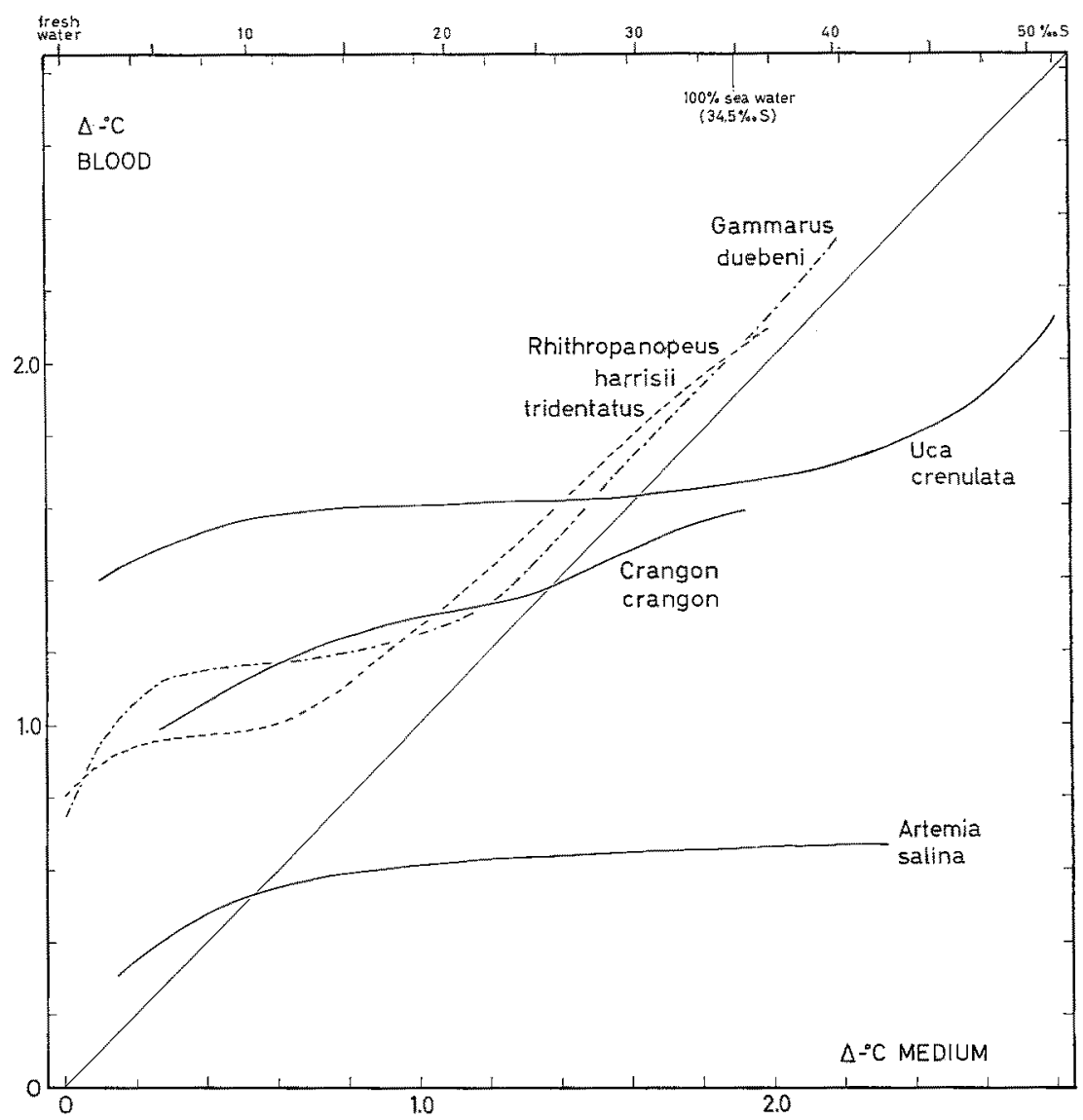

Abb. 6: Beispiele der Leistungen eurysaliner Osmoregulierer nach längerem Aufenthalt in verschiedenen Salinitäten (Süßwasser bis etwa $50 \%$ ). Angegeben ist jeweils die osmotische Gesamtkonzentration der Blutflissigkeit. $G$, duebeni und $R$. barrisii iridentatus sind hyperosmotische Regulierer; ihr Blut ist hyperosmotisch in verdünntem Meerwasser aber mehr oder minder isosmotisch in darüberliegenden Salinitäten. U. crenulata, $C$. crangon und $A$. salina sind hyper-hypoosmotische Regulierer; ihr Blut ist hyperosmotisch in verdünntem Meerwasser und hypoosmotisch in darïberliegenden Salinitäten. (Nach verschiedenen Autoren, aus KrNNE 1963b)

\& Cragg 1940a), G. duebeni (Beadle \& Cragg 1940a, Kinne 1952) G. oceanicus, Marinogammarus finmarchicus (Werntz in Prosser \& Brown 1961), Carcinus maenas (Duval 1925, Schlieper 1929a, b, Nagel 1934), Rhithropanopeus harrisii tridentatus (KInne \& Rotthauwe 1952), Palaemonetes varians, Palaemon serratus, Palaemon squilla, Panaeus indicus, P. carinatus, Metapenaeus dobsoni (Panik kar 1939, $1940,1941,1950)$, Metapenaeus monoceros (PanikKar \& Viswanathan 1948), Uca 
crenulata (Jones 1941), Crangon crangon (Broekema 1941, Flügel 1959), Artemia salina (Croghan 1958a, b), Pachygrapsus crassipes (Jones 1941); die Fische Gasterosteus (Gueylard 1924, Koch \& Heuts 1942, 1943 und spätere Arbeiten), Anguilla (Callamand 1943), verschiedene Cyprinodonten und viele andere (Krogh 1939,

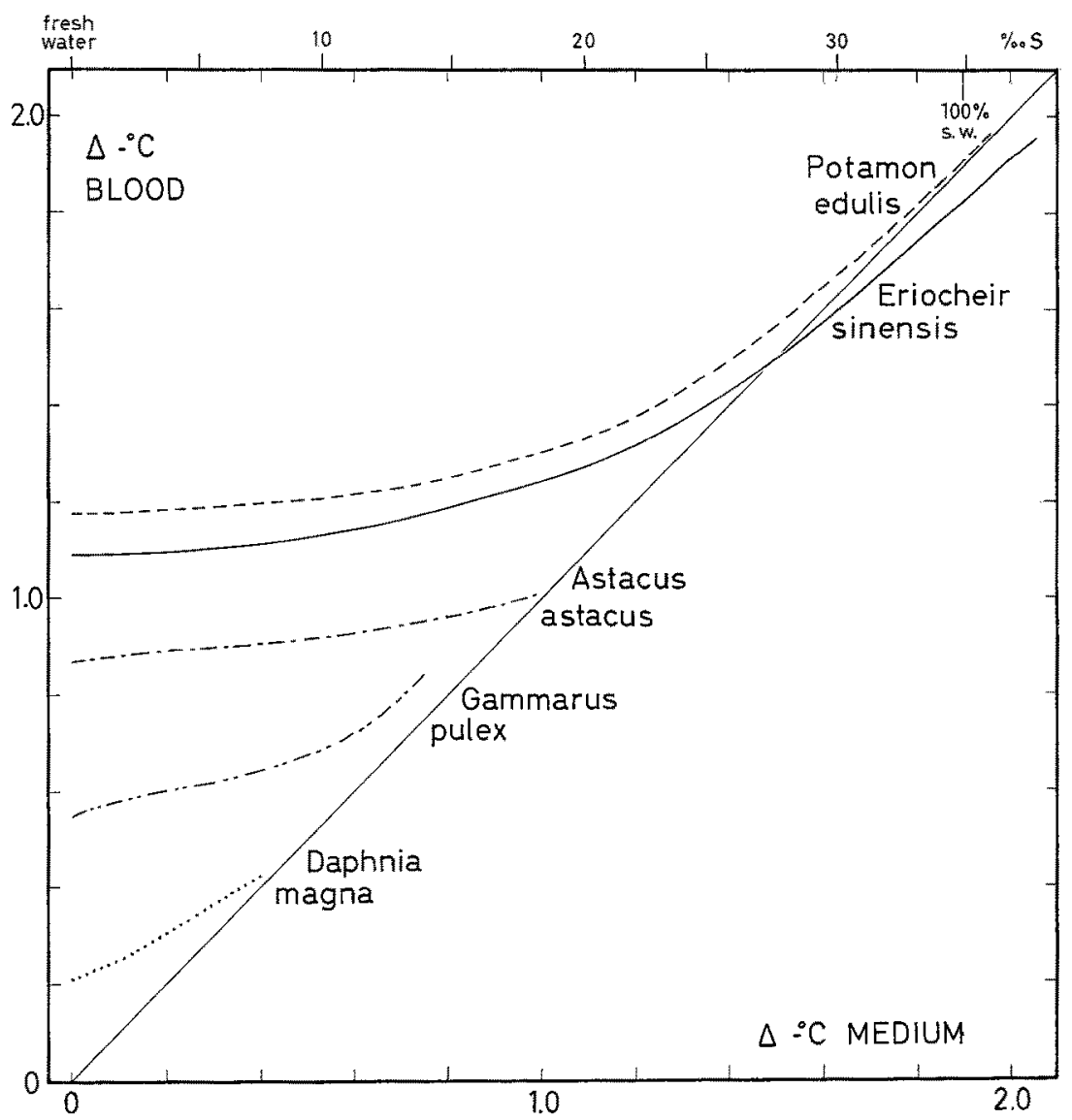

Abb. 7: Beispiele der Leistungen (1) eines holeurysalinen Regulierers (E. sinensis) und (2) mehrerer oligosaliner Osmoregulierer ( $P$. edulis, A. astacus, $G$. pulex und $D$. magna). (Nach verschiedenen Autoren, aus KINNE 1963b)

Black 1957, Pearse \& Gunter 1957, Nicol 1960, Prosser \& Brown 1961). Holeurysaline Osmoregulierer (Abb. 7) können in ausreichendem Maße regulieren in Salzkonzentrationen, welche von reinem Süßwasser bis zu reinem Meerwasser und darüber hinaus reichen. Beispiele sind: die Wollhandkrabbe Eriocheir sinensis (Scholles 1933, Schwabe 1933, Schlieper 1935, Кoch 1954, Косн \& Evans 1956a, b); die Fische Cyprinodon macularius und Fundulus beteroclitus; vermutlich gehören hierher auch verschiedene Turbellarien, Rotatorien und Oligochaeten (vgl. KINNE 1964b). Oligosaline Osmoregulierer können in ausreichendem Maße nur in reinem Süßwasser regulieren; ihr osmoregulatorischer Apparat bricht schon bei Salz- 
gehalten von wenigen Promille zusammen. Die Körperflüssigkeiten oligosaliner Osmoregulierer sind gegenïber dem umgebenden Süßwasser erheblich hyperosmotisch. Zu dieser Gruppe gehören die meisten typischen Süßwasserbewohner (Abb. 7).

Unter den Fischen nehmen die Elasmobranchier und die osmolabilen Myxinoiden insofern eine Sonderstellung ein, als ihr Blut weitgehend isosmotisch mit dem umgebenden Meerwasser ist. Der eigentliche Gehalt an Blutsalzen liegt jedoch bei den Elasmobranchiern im allgemeinen erheblich unter dem Salzgehalt des Meerwassers und unterscheidet sich kaum von dem hypoosmotischer mariner Knochenfische. Die Differenz $z$ wischen den Osmokonzentrationen von Außen- und Innenmedium wird durch den hohen Harnstoffgehalt des Blutes ausgeglichen, Sobald eurysaline Elasmobranchier in den Bereich eines Ästuariums gelangen, reduzieren sie den Ureagehalt ihres Blutes und erniedrigen ihre Innenkonzentration dadurch rasch und wirkungsvoll.

Die physiologischen Mechanismen der Osmoregulation waren und sind Gegenstand zahlreicher Untersuchungen. Das Augenmerk der Untersucher hat sich vor allem auf die größeren Formen konzentriert, insbesondere auf die Crustaceen und Pisces. In diesen beiden Gruppen erwiesen sidh die Regulationsmechanismen als redht komplex; in vielen Fällen sind mehrere Organe, welche unter endokriner oder nervöser Kontrolle stehen können, beteiligt. Die hauptsächlichsten Osmoregulationsorgane sind Kiemen (häufig verantwortlich für den Salzhaushalt), Darm (häufig verantwortlich für den Wasserhaushalt), Nephridialkanäle sowie Antennendrüsen (bei einigen Krebsen); vor allem bei morphologisch weniger differenzierten Lebensformen, wie Protozoen und Cnidariern, sind offenbar die Körperoberfläche und deren Permeabilitätsverhältnisse von besonderer Bedeutung. Ausführliche Reviews über die osmoregulatorischen $\mathrm{Me}-$ chanismen der Crustaceen sind von Robertson (1957, 1960a), Nicol (1960), Shaw (1960), Prosser \& Brown (1961), Lockwood (1962) und Kinne (1963b, 1964b) publiziert worden.

\section{Adaptationen}

Unter den Kompensationsmöglichkeiten der ästuarinen Organismen sind die Adaptationen von besonderem Interesse. Sie unterscheiden sich von den Regulationen im wesentlichen dadurch, daß sie echte funktionelle oder strukturelle Veränderungen im Organismus verursachen, Zeit zu ihrer "Entwicklung " benötigen und sich vielfach nicht auf spezifische Organe beschränken, sondern mehr oder minder alle Ebenen der organismischen Organisation betreffen. Regulationen dagegen haben im allgemeinen die Wiederherstellung des "Sollzustandes" aber keine echten Veränderungen des Status quo ante zur Folge; sie laufen meist in Minuten oder Stunden - also recht schnell ab und sind das Ergebnis der routinemäßigen Aktivität spezifischer, präexistierender Reaktionssysteme. Selbstverständlich können Regulationen und Adaptationen in einem unter Stress stehenden Organismus gleichzeitig ablaufen; vielfach lassen sie sich auch nicht bis in alle Einzelheiten unterscheiden. Nichtsdestoweniger erscheint aber für den Experimentator eine begriffliche Differenzierung im Interesse einer klaren Analyse der konstatierten Reaktionen erforderlich.

Eine Adaptation ist ein ökologisches Phänomen. Der Begriff „Adaptation“ bezeichnet eine Neueinstellung lebender Systeme im Anschluß an Veränderungen in den 
Intensitätsmustern von Umweltfaktoren, welche letztlich zu einer relativen Erhöhung der Überlebens-, Vermehrungs- oder Konkurrenzkapazität der betroffenen Individuen führt und somit objektiv meßbare potentielle Vorteile im existenzökologischen Sinne beinhaltet (KINNE 1963b, 1964a, c). Das Nettoergebnis einer Adaptation ist eine Kompensation ungünstiger Umwelteinwirkungen, die vielfach einhergeht mit einer $\mathrm{Zu}$ nahme der inneren Stabilität und Homoeostasis. Neuere Reviews und kritische Erörterungen über das Problem der Adaptation sind von AdolPH (1964), PrECHT (1964), Prosser (1964) und KinNe (1964a, b, c) verfaßt worden. Der interessierte Leser sei in diesem Zusammenhang besonders auch auf die umfassende Neuerscheinung Handbook of Pbysiology, Section 4, 1964, hingewiesen.

Die Adaptationen ästuariner Organismen bestehen vermutlich in den meisten Fällen sowohl aus einer genetischen als auch aus einer nichtgenetischen Komponente. Genetische Adaptationen beruhen letztlich auf Veränderungen im Genotyp, welche der besonderen Umweltsituation Rechnung tragen und sich sowohl in quantitativen als auch qualitativen Abänderungen (besonders der elementaren biochemischen und physiologischen Prozesse und der primären Proteinstrukturen) manifestieren. Sie setzen genetische Variabilität voraus und kommen offenbar im Verlaufe von Selektionsprozessen zustande. Dem Zugriff des Experimentators ist der Problemkreis der genetischen Adaptation nur schwer zugänglich. Obgleich die Vermutung naheliegt, daß gerade die mit der Euryplastizität ästuariner Organismen im Zusammenhang stehenden physiologisch-ökologischen Aspekte des Lebens in Ästuarien auf langfristige genetische Adaptationen zurückgehen, lassen die uns gegenwärtig zur Verfügung stehenden, unzureichenden Informationen eine ausführliche Behandlung nicht zu.

Nichtgenetische Adaptationen sind auch unter den Bezeichnungen Akklimatisationen und Akklimationen bekannt. Sie beschränken sich auf unmittelbar von der Umwelt induzierte Veränderungen, die als solche nicht an die nachfolgenden Generationen weitergegeben werden. Nichtgenetische Adaptationen sind primär quantitativer Natur; sie können funktionelle und strukturelle Modifikationen auf der Ebene subzellulären und zellulären Geschehens ebenso beinhalten wie Veränderungen in Geweben und Organen oder in der organismischen Integration. Zu ihrer Erhaltung bedürfen die reversiblen Akklimatisationen ständiger Reengagements durch den wirksamen Umweltstress.

Potential und Mechanismus einer nichtgenetischen Adaptation sind Produkte der Evolution und repräsentieren genetisch fixierte Eigenarten. Unter diesem Blickwinkel kann man daher genetische und nichtgenetische Adaptationen auch als zwei. Aspekte ein und desselben Problemkreises betrachten.

Nichtgenetische Adaptationen spielen für das Leben in Astuarien offenbar eine wesentliche Rolle. Bei vielen Astuarbewohnern scheint die Fähigkeit zur Akklimatisation an die Inkonstanz der Lebensbedingungen, die ständigen Intensitätsschw ankungen hochwirksamer Umweltfaktoren, mindestens ebenso wichtig zu sein wie die Fähigkeit zur Anpassung an extreme, relativ konstante Faktorintensitäten. Leider basieren unsere Kenntnisse über nichtgenetische Adaptationen nahezu ausschließlich auf experimentellen Studien, die unter konstanten Salinitäts- oder Temperaturintensitäten durchgeführt worden sind. Hier sind baldmöglichst weitere Untersuchungen unter fluktuierenden Umweltbedingungen sehr erwünscht. Es wäre zum Beispiel zu klären, 
inwieweit ein mit Unterbrechungen zur Wirkung kommender Stress die gleichen oder ähnlichen Akklimatisationen bewirken kann wie ein kontinuierlich einwirkender (siehe hierzu auch Adolph 1956).

Viele der bisherigen Untersuchungen basieren auf monofaktoriellen Analysen auf Versuchsbedingungen also, bei denen nur ein einziger Umweltfaktor verändert wurde. Derartige Situationen kommen in Astuarien praktisch nicht vor. Es erscheint daher dringend notwendig, zukünftige Akklimatisationsversuche auch unter Umweltbedingungen durchzuführen, die den natürlichen Gegebenheiten zumindest ähnlich sind. Von solchen Versuchsanordnungen dürfen wir Ergebnisse erwarten, die uns tiefere Einblicke in die ökologische Bedeutung der Akklimatisationserscheinungen gestatten.

Uber nichtgenetische Adaptationen an Salinitäts- und Temperaturveränderungen habe ich im einzelnen erst kürzlich in zusammenfassenden Arbeiten berichtet (KINNE $1963 a, 1964 a, b, c)$. Ich kann mich hier daher kurz fassen. Akklimatisationen an veränderte Salinitätsbedingungen scheinen sich vor allem auf den Wasser- und Salzhaushalt der betroffenen Individuen auszuwirken. Die einfachsten und phylogenetisch ältesten Anpassungserscheinungen beruhen auf einer generellen Gewebeakklimatisation, etwa bei den Osmokonformisten, denen ja spezifische Regulationsorgane fehlen. Bei Osmoregulierern verlagert sich der Schwerpunkt der Anpassungen häufig in die unmittelbaren Bereiche der beanspruchten Regulationsorgane (Kiemen, Darm, Exkretionszentren).

Die Mechanismen der Temperaturadaptation sind noch weitgehend unbekannt. Eine Zunahme der Temperaturresistenz scheint letztlich auf Verschiebungen im Bereich intermolekularer Kräfte zurückzugehen, welche ihrerseits die Stabilisierung protoplasmatischer Komponenten beeinflussen. In vielen Fällen ist eine Zunahme der Kälte- und Hitzeresistenz nicht nur mit einer Zunahme der protoplasmatischen Stabilität verbunden, sondern auch mit einer relativen Abnahme der biochemischen Aktivität. Mit einer Resistenzerhöhung gegenüber kritischen Temperaturintensitäten geht übrigens häufig eine ganz allgemeine, unspezifische Erhöhung der Widerstandsfähigkeit gegenüber Umweltstressen einher. So kann eine Akklimatisation an extreme Temperaturen beispielsweise zu einem gleichzeitigen Anstieg der Resistenz gegenüber niedriger Sauerstoffspannung, schädigenden Strahlungen oder chemischen Zellgiften führen.

Nichtgenetische Adaptationen an veränderte Salzgehalts- oder Temperaturbedingungen manifestieren sich besonders offensichtlich in Verschiebungen der Letalgrenzen sowie in Veränderungen der Intensität und Okonomie der Stoffwechselprozesse (Atmung, Regulation, Wachstum, Fortpflanzung) und der Aktivität; gleichzeitig können sie sich auch auf verhaltensphysiologische und strukturelle Aspekte erstrecken. Für ästuarine Organismen - insbesondere jene in den gemäßigten Zonen - spielt die Fähigkeit zur Adaptation an veränderte Temperaturverhältnisse ganz offensichtlich eine besonders wichtige Rolle, da spezifische Regulationsorgane fehlen. Zur Kompensation ungünstiger Salinitätsveränderungen steht den meisten Ästuarbewohnern dagegen, wie wir gesehen haben, eine Reihe von Regulationsmöglichkeiten zur Verfügung.

Die Geschwindigkeit, mit der eine bestimmte nichtgenetische Adaptation erworben werden kann, hängt von mehreren Faktoren ab; der untersuchten Art, der als Kriterium dienenden Lebensäußerung, dem Lebensalter (die Geschwindigkeit nimmt meist mit zunehmendem Alter ab), der Stoffwechselintensität (meist Verlangsamung mit abneh- 
mender Intensität), dem physiologischen und ontogenetischen Zustand des Versuchstieres und schließlich dem Ausmaß und der Art der Umweltveränderung. Soweit hier eine Verallgemeinerung möglich ist, läßt sich sagen, daß Akklimatisationen bei ästuarinen Wirbellosen und Fischen Tage bis Woden dauern. Im Hinblidk auf deren Lebenserwartung mögen solche Zeitspannen als recht lang erscheinen. Rasche Neueinstellungen können jedoch zweifellos auch nachteilig sein: sie könnten den Organismus leicht der Gefahr einer endlosen Serie von "An - aus“-Reaktionen aussetzen; der neue stationäre Zustand jeweils höchstmöglicher Stoffwechselökonomie würde dann praktisch nie erreicht werden.

\section{DISKUSSION}

Die spezifischen, recht lebensfeindlichen Umweltbedingungen in den Astuarien bieten nur einer relativ kleinen Gruppe von Lebensformen eine Existenzmöglichkeit. Die Vertreter dieser Gruppe zeichnen sich, trotz ihrer zum Teil erheblichen morphologischen und organisatorischen Unterschiede, durch weitgehend ähnliche physiologische und ökologische Eigenarten aus. Insbesondere ist den Ästuarbewohnern eine Reihe von Kompensationsmöglichkeiten gemeinsam. Diese Kompensationsmöglichkeiten lassen sich in vier Aspekte unterteilen: Ausweichreaktionen, Kontaktverminderungen, Regulationen und Adaptationen. Es muß hier ausdrücklich vermerkt werden, daß besonders die letzteren drei Aspekte gleichzeitig in ein und demselben Individuum wirksam werden können und auch hinsichtlich ihrer Wirkungen und Mechanismen nicht immer bis ins letzte Detail zu trennen sind. Eine Unterteilung in die genannten vier Aspekte macht es aber möglich, die komplexen und vielschichtigen physiologisch-ökologischen Reaktionen, über welche in der Fachliteratur berichtet worden ist, in einem geordneten Gedankengebäude zu präsentieren. Sie könnte auch bei weiteren Analysen als brauchbares Werkzeug dienen.

Ausweichreaktionen sind als Kompensationsmöglichkeiten vermutlich sehr verbreitet. Die Wirkungen ungünstiger Salinitäten können durch derartige Realstionen ebenso vermindert werden wie diejenigen der Temperatur. Kontaktverminderungen sind vor allem zur Kompensation plötzlicher, starker Salzgehaltsveränderungen geeignet; temporären Extremtemperaturen gegenüber sind sie weitaus weniger wirkungsvoll, wenn man von der Transformation in Ruhestadien absieht. Auch die verschiedenen Regulationsmechanismen sind ganz auf den Salinitätsfaktor abgestellt. Eine wirkungsvolle Temperaturregulation fehlt vollkommen. Nichtgenetische Adaptationen spielen sowohl bezüglich der Salinität als auch der Temperatur eine Rolle. Allerdings liegen zur Zeit über Salzgehaltsakklimatisationen wesentlich weniger Ergebnisse vor als über Temperaturakklimatisationen. Wir dürfen hier in den nächsten Jahren noch wichtige neue Informationen erwarten.

Insgesamt ergibt sich also, daß hinsichtlich der Kompensation ungünstiger Salinitätsverhältnisse Ausweichreaktionen, Kontaktverminderungen und Regulationen (Ionen-, Volum-, Osmoregulation) eine besondere Rolle spielen. Bei ungünstigen Temperaturverhältnissen dagegen beschränken sich die Kompensationsmöglichkeiten ror allem auf Ausweichreaktionen und Adaptationen. Astuarine Organismen können 
demnach den schädigenden Wirkungen extremer Salinitätsverhältnisse schneller und effektvoller begegnen.

Ich habe in meinen Ausführungen mehrfach darauf hingewiesen, daß eine erhöhte Variabilität der Lebensbedingungen ein Hauptcharakteristikum des Ästuarbereiches darstellt. Nichtsdestoweniger ist aber gerade das Vorhandensein einer solchen erhöhten Variabilität, phylogenetisch gesehen, ein recht konstanter Faktor. Diese historische Permanenz ästuariner Variabilität und Instabilität hat zu Spekulationen Anlaß gegeben, nach denen die wenigen Arten, welche sich im ästuarinen Lebensraum behaupten konnten, phylogenetisch alte und recht konservative Formen darstellen. Nach EMERY et al. (1957) ist zum Beispiel gerade die Euryplastizität der ästuarinen Organismen ein genetisches Merkmal, welches offenbar nur schwer zu erwerben ist, das aber einmal vorhanden - im Sinne der Evolution sehr konservativ ist. Auch im Bereich der Ontogenie scheint Euryplastizität vielfach erkauft zu sein durch eine relativ niedrige Lebensintensität und eine gewisse "Schwerfälligkeit". So liegen Stoffwechselrate, Aktivität und Vermehrungspotential euryplastischer Arten gewöhnlich weit unter den Vergleichswerten ihrer empfindlicheren stenoplastischen Verwandten.

\section{ZUSAMMENFASSUNG}

1. Die physiologischen und ökologischen Eigenarten ästuariner Organismen werden erörtert, und zwar unter besonderer Berücksichtigung der diesen zu Gebote stehenden Kompensationsmöglichkeiten gegenüber unerwünschten Salinitäts- und Temperaturverhältnissen. Trotz ihrer unterschiedlichen Baupläne zeichnen sich typische Astuarbewohner durch eine Reihe recht ähnlicher Reaktionsmuster aus.

2. Permanente Ästuarbewohner sind in ihren physiologischen und ökologischen Reaktionen durchweg ausgesprochen euryplastisch. Sie verfügen über ein großes abiotisches Potential. Ihr biotisches Potential (Widerstandsfähigkeit gegenüber biotischen Faktoren; Konkurrenzvermögen) dagegen ist vielfach gering.

3. Es werden vier Gruppen von Kompensationsmöglichkeiten unterschieden: Ausweichreaktionen (direkte und indirekte), Kontaktverminderungen (Absonderung schützender Substanzen, Rückzug in Wohnhöhlen etc., Verringerung des Oberflächen/ Volumen-Quotienten, Einziehen empfindlicher Organe, Schalenverschluß, langfristige Veränderungen der Körperform, Transformationen in Ruhestadien), Regulationen (Ionen-, Volum- und Osmoregulation), Adaptationen (genetische und nichtgenetische).

4. Die vier unterschiedenen Kompensationsmöglichkeiten lassen sich nicht immer bis ins letzte Detail klar voneinander trennen. Sie können gleichzeitig in ein und demselben Individuum wirksam werden. Ihre begriffliche Differenzierung erleichtert aber die Erarbeitung der teilweise recht komplexen Zusammenhänge.

5. Hinsichtlich einer Kompensation der schädlichen Wirkungen ungeeigneter Salinitätsverhältnisse sind Ausweichreaktionen, Kontaktverminderungen und Regulationen von besonderer Bedeutung. Bei ungünstigen Temperaturverhältnissen beschränken sich die Kompensationsmöglichkeiten vor allem auf Ausweichreaktionen und Adaptationen. Ungünstige Salzgehaltsverhältnisse können demnach rascher und effektvoller kompensiert werden. 
6. Das profilierteste physio-ökologische Merkmal der Ästuarbewohner, die Euryplastizität, scheint eine schwer erwerbbare, phylogenetisch recht alte und konservative Eigenschaft darzustellen.

\section{ZITIERTE LITERATUR}

Ackerman, E., 1962. Biophysical science. Prentice-Hall, Inc., Englewood Cliffs, N. J., 626 pp. Adolph, E. F., 1956. General and specific characteristics of physiological adaptations. Am.J. Physiol. 184, 18-28.

- 1964. Perspectives of adaptation: some general properties. In: Handbook of physiology. Ed. by D. B. Drll, E. F. Adolph \& C. G. Wrlber, Am. Physiol. Soc., Wash., Sect. 4, 27-35.

Alexander, W. B., Southgate, B. A. \& Bassindale, R., 1935. Survey of the River Tees. Part. 2. The estuary - demical and biological. Tech. Paper Dept. Sci. Indust. Res. Water Pollution Res. No. 5, 1-171.

BeAdLE, L. C., 1931. The effect of salinity changes on the water content and respiration of marine invertebrates. J. exp. Biol. 8, 211-227.

- 1934. Osmotic regulation in Gunda ulvae. J. exp. Biol. 11, 382-396.

- 1937. Adaptation to changes of salinity in the polychaetes. I. Control of body volume and of body fluid concentration in Nereis diversicolor. J. exp. Biol. 14, 56-70.

- 1957. Comparative physiology: osmotic and ionic regulation in aquatic animals. A. Rev. Pbysiol. 19, 329-354.

- \& CRAGG, J. B., 1940. Studies on adaptation to salinity in Gammarus spp. 1. Regulation of blood and tissues and the problem of adaptation to fresh water. J. exp. Biol. 17, 153-163.

BERGQUisT, P. L., 1958a. Evidence for separate mechanisms of sodium and potassium regulation in Homosira banksii. Physiologia Pl. 11, 760-770.

- 1958b. Effect of potassium cyanide on apparent free space in a brown alga. Nature, Lond. $181,1270$.

Bethe, A., Holst, E. von \& Huf, E., 1935. Die Bedeutung des medhanischen Innendrucks für die Anpassung gepanzerter Seetiere an Anderungen des osmotischen Außendrucks. Pflïgers Arch. ges. Physiol. 235, 330-334.

Biebl, R., 1937. Okologische und zellphysiologische Studien an Rotalgen der englischen Südküste, Beih. bot. Zbl. (A) 57, 381-424.

- 1956. Zellphysiologish-ökologische Untersuchungen an Enteromorpha clatbrata (Roth) Greville. Ber. bot. Ges. 69, 75-86.

BLACK, V. S., 1957. Excretion and osmoregulation. In: The physiology of fishes. Ed. by M. E. Brown. Acad. pr., New York, Vol. 1, 447 pp.

Burnss, L. R., 1951. Physiology and biochemistry of algae. In: Manual of phycology. Ed. by G. M. SмLтн. Chronica Bot. Co., Waltham, Mass., 263-291.

Braarud, T., 1951. Salinity as an ecological factor in marine phytoplankton. Pbysiologia Pl. 4, $28-34$.

- \& PAPras, I., 1951. Experimental studies on the dinoflagellate Peridinium triquetrum(Ehrb.) Lebour. Avb. norske Vidensk Akad. Oslo (Mat. nat. Kl.) 1951 (2), 1-23.

Broekema, M. M. M., 1941. Seasonal movements and the osmotic behaviour of the shrimp, Crangon crangon L. Archs néerl. Zool. 6, 1-100.

Brown, R. \& DanieliI, J. F. (Eds.), 1955. Active transport and secretion. Symp. Soc. exp. Biol. 8, 1-516.

Buchrerm, A., 1915. Der Einfluß des Außenmediums auf den Turgordrudk einiger Algen. Mitt. naturf. Ges. Bern, 70-113.

Bünning, E., 1934. Zellphysiologische Studien an Meeresalgen. Protoplasma 22, 444-456.

CAllamand, O., 1943. L'anguille européenne (Anguilla anguilla L.) les bases physiologiques de sa migration. Annls Inst. océanogr., Monaco 21, 361-440.

CASPERS, H., 1948. Okologische Untersuchungen über die Wattentierwelt im Elbeästuar. Zool. Anz. (Suppl. Bd.) 13, 350-359.

- 1955. Limnologie des Elbeästuars. Verh. int. Ver. Limnol. 12, 613-619. 
- 1958. Biologie der Brackwasserzonen im Elbeästuar. Verh. int. Ver. Limnol. 13, 687-698.

- 1959. Die Einteilung der Brackwasser-Regionen in einem Âstuar. Arcbo Oceanogr. Limrol. (Suppl.) 11, 153-169.

Clarke, F. W., 1924. The data of geochemistry. Bull. U.S. geol. Surv. 770, $841 \mathrm{pp}$.

COLE, W. H., 1940. The composition of fluids and sera of some marine animals and of the sea water in which they live. J. gen. Physiol. 23, 575-584.

Collander, R., 1939. Permeabilitätsstudien an Charazeen. 3. Die Aufnahme und Abgabe von Kationen. Protoplasma 33, 215-257.

Croghan, P. C., 1958a. The survival of Artemia salina (L.) in various media. J. exp. Biol. 35, 213-218.

- 1958b. The osmotic and ionic regulation of Artemia salina (L.). J.exp. Biol. 35, 219-233.

DAY, J. H., 1951. The ecology of South African estuaries. 1. A review of estuatine conditions in general. Trans. R. Soc. S. Afr. 33, 53-91.

Drevs, P., 1896. Die Regulation des osmotischen Druckes in Meeresalgen bei Schwankungen des Salzgehaltes im Außenmedium. Arch. Ver. Freunde Naturg. Meckenb. 49, 91-135.

Droop, M. R., 1958. Optimum relative and actual ionic concentrations for growth of some euryhaline algae. Verh. int. Ver. Limnol. 13, 722-730.

Duval, M., 1925. Récherches physico-chimiques et physiologiques sur le milieu intérieur des animaux aquatiques. Modifications sous linfluence du milieu extérieur. Annls Inst. océanogr., Monaco 2, 233-403.

ELLIS, W. G., 1937. The water and electrolyte exchange of Nereis diversicolor (Mueller). J. exp. Biol. 14, 340-350.

- 1939. Comparative measurements of water and electrolyte exchange in a stenohaline and a euryhaline polychaete. J. exp. Biol. 16, 483-486.

Emery, K. O., Stevenson, R. E. \& Hedgpeth, J. W., 1957. Estuaries and lagoons. Mem. geol. Soc. Am. 67 (1), 673 749.

Epprex, R. W., 1958a. Sodium exclusion and potassium retention by the red marine alga, Porphyra perforata. J. gen. Physiol. 41, 901-911.

- 1958b. Potassium-dependent sodium extrusion by cells of Porpbyra perforata, a red marine alga. J. gen. Physiol. 42, 281-288.

- 1962. Major cations. In: Physiology and biochemistry of algae. Ed. by R. A. LEwIN, Acad. pr., New York, 255-266.

- \& Bovell, C. R., 1958. Sulfuric acid in Desmarestia. Biol. Bull mar. biol. Lab., Woods Hole 115, 101-106.

- \& Crrus, C. C., 1960. Cation regulation and survival of the red alga, Porphyra perforata, in diluted and concentrated sea water. Biol. Bull. mar, biol. Lab., Woods Hole 118, 55-65.

Fi.üGrI, H., 1959. Zum Problem der Osmoregulation im tropischen Brackwasser. Naturweissenschaften 46, 213.

FRETTER, V., 1955. Uptake of radioactive sodium (24Na) by Nereis diversicolor Mueller and Perinereis cultrifera Grube. J. mar. biol. Ass. U.K. 34, 151-160.

GARMS, R., 1961. Biozönotische Untersuchungen an Entwässerungsgräben in Flußmarschen des Elbeästuars. Arch. Hydrobiol. (Suppl.) 26, 344-462.

GueYLARD, F., 1924. Paris, Ph. D. Thesis (quoted in BlAck, 1957).

- 1925. De l'adaptation aux changements de salinité. Recherches biologiques et physcochimiques sur l'épinoche (Gasterostens leinus Cur. et Val.). Archs Phys. biol. 3, 79-187.

Gullard, R. R. L., 1962. Salt and osmotic balance. In: Physiology and biochemistry of algae. Ed. by. R. A. LEwin, Acad. pr., New York, 529-540.

- \& Rrther, J. H., 1962. Studies of marine planktonic diatoms. 1. Cyclotella nana Hustedt, and Detonula confervacea (Cleve) Gran. Can. J. Microbiol. 8, 229-239.

Handbook of physiology. Sect. 4. Adaptation to the environment (1964). Ed. by D. B. Dill, E. F. Adolph \& C. G. Wilber. Am. Physiol. Soc., Washington, 1056 pp.

HöFLER, K., 1931. Hypotonietod und osmotische Resistenz einiger Rotalgen. Öst. bot. Z. 80, 51-71.

- 1932. Plasmolyseformen bei Chaetomorpha und Cladophora. Protoplasma 16, 189-214.

Hope, A. B. \& Walker, N. A., 1960. Ionic relations of Chara australis. 3. Vacuolar fluxes of sodium. Aust. J. biol. Sci. 13, 276-291. 
JoNEs, L. L., 1941. Osmotic regulation in several crabs of the Pacific coast of North America. J. cell. comp. Pbysiol. 18, 79-92.

JørGensen, C. B. \& Dales, R. P., 1957. The regulation of volume and osmotic regulation in some nereid polychaetes. Physiologia comp. Oecol. 4, 357-374.

JørGensen, E. G., 1960. The effects of salinity, temperature, and light intensity on growth and dilorophyll formation of Nitzschia ovalis. Yb. Carnegie Inst. Wash. 59, 348-349.

Kesseler, H., 1959. Mikrokryoskopische Untersuchungen zur Turgorregulation von Cbaetomorpha linum. Kieler Meeresforsch. 15, 51-73.

- 1960. Morphologische und zellphysiologische Untersuchungen von Chaetomorpha linum. Helgol. Wiss. Meeresunters. 7, 114-124.

- 1962. Beziehungen zwischen Atmung und Turgorregulation von Chaetomorpha linum. Helgol. Wiss. Meeresunters. 8, 243-256.

- 1964. Die Bedeutung einiger anorganischer Komponenten des Seewassers für die Turgorregulation von Chatomorpha linum (Cladophorales). Helgol. Wiss. Meeresunters. 10, 73-90.

KrnNe, O., 1952. Zur Biologie und Physiologie von Gammarus duebeni Lillj. V: Untersuchungen über Blutkonzentration, Herzfrequenz und Atmung. Kieler Meeresforsch. 9, 134-150.

- 1956. Uber Temperatur und Salzgehalt und ihre physiologisch-biologische Bedeutung. Biol. Zbl. 75, 314-327.

- 1963a. The effects of temperature and salinity on marine and brackish water animals. 1. Temperature. Oceanogr. mar. Biol. A. Rev. 1, 301-340.

- 1963b. Adaptation, a primary mechanism of evolution. In: Phylogeny and evolution of Crustacea. Ed. by H. B. WhITTINGToN \& W. D. I. Rolfe. Mus. comp. Zool. Harvard Coll., Cambridge, Mass. (Spec. publ.), 27-50.

- 1964a. Animals in aquatic environments: crustaceans. In: Handbook of physiology. Ed. by D. B. Dili, E. F. Adolph \& C. G. Wirber. Am. Physiol. Soc., Wash., Sect. 4, 669-682.

- 1964b. The effects to temperature and salinity on marine and brackish water animals. 2. Salinity and temperature-salinity combinations. Oceanogr. mar. Biol. A. Rev. 2, 281-339.

- 1964c. Non-genetic adaptation to temperature and salinity. Helgol. Wiss. Meeresunters. 9, $433-458$.

- \& RotThauwe, H.-W., 1952. Biologische Beobachtungen und Untersuchungen über die Blutkonzentration an Heteropanope tridentatus Maitland (Decapoda). Kieler Meeresforscb. 8, 212-217.

K.tTchnng, J. A., 1938. Contractile vacuoles. Biol. Rev. 13, 403-444.

- 1948. The physiology of contractile vacuoles. 6. Temperature and osmotic stress. J. exp. Biol. 25, 421-436.

- 1951. The physiology of contractile vacuoles. 7. Osmotic relations in a suctorian, with special reference to the mechanism of control of vacuolar output. J. exp. Biol. 28, 203-214.

- 1952. Contractile vacuoles. Symp. Soc. exp. Biol. 6, 145-146.

- 1954. Osmoregulation and ionic regulation in animals without kidneys. Symp. Soc. exp. Biol. 8, 63-75.

Kock, H. J., 1954. Cholinesterase and active transport of sodium chloride through the isolated gills of the crab Eriocheir sinensis (M. Edw.). In: Recent developments in cell physiology. Ed. by J. A. Kitching, Acad. pr., New York, 15-27.

- \& Evans, J., 1956a. On the absorption of sodium from dilute solutions by the crab Eriocheir sinensis (M. Edw.). Meded. K. vlaam. Acad. 18, 3-15.

- - 1956b. Influence of a basic dye, thionine, on the absorption of sodium by the crab Eriocheir sinensis (M. Edw.). Meded. K. vlaam. Acad. 18, 3-11.

- \& Heurs, M. J., 1942. Influences de l'hormone thyroidieme sur la regulation osmotique chez Gasterostevis aculeatus L. forme gymnurus Cuv. Annls Soc, r. zool. Belg. 73, 165-172.

- 1943. Osmotic regulation, sexual cycle and migration of reproduction of the épinoches. Archs int. Physiol. 53, 253-266.

Koller, G., 1939. Uber die Nephridien von Physcosoma japonicum. Zool. Anz. (Suppl. Bd.) $12,440-447$.

KötTer, F., 1961. Die Pflanzengesellschaften im Tidegebiet der Unterelbe. Arch. Hydrobiol. (Suppl.) 26, 106-184.

Kotrie, P., 1961. Hydrobiologie der Oberelbe. Arch. Hydrobiol. (Suppl.) 26, 221-343. 
Krishnamoortiri, B., 1951. Studies on the osmotic properties of the eggs and larvae of a brackish-water polychaete, Marphysa gravelyi Southern. Proc. Indian Acad. Sci. (Sect. B) 34, 199-209.

KroGF, A., 1938. The salt concentration in the tissues of some marine animals. Skand. Arch. Pbysiol. 80, 214-222.

- 1939. Osmotic regulation in aquatic animals. Cambridge Univ. pr., London, 242 pp.

Lewrin, R. A. (Ed.), 1962. Physiology and biochemistry of algae. Acad. pr., New York, 929 pp.

- \& Gulllard, R. R. L., 1963. Diatoms. A. Rev. Microbiol. 17, 373-414.

Lockwood, A. P. M., 1962. The osmoregulation of Crustacea. Biol. Rev. 37, 257-305.

MacRobire, E. A. C. \& Dainty, J., 1958a. Sodium and potassium distribution and transport in the seaweed Rbodymenia palmata (L.) Grev. Physiologia Pl. 11, 782-801.

,--1958 b. Ion transport in Nitellopsis obtusa. J. gen. Physiol. 42, 335-353.

McСombie, A. M., 1960. Actions and interactions of temperature, light intensity and nutrient concentration on the growth of the green alga, Cblamydomonas reinhardi Dangeard. $J$. Fish. Res. Bd. Can. 17, 871-894.

Mǜlzer, R., 1936. Die osmoregulatorische Bedeutung der kontraktilen Vakuolen von Amoeba proteus, Zoothamnium biketes und Frontonia marina. Arch. Protistenk. 87, 345-382.

Nagex, H., 1934. Die Aufgaben der Exkretionsorgane und der Kiemen bei der Osmoregulation von Carcinus maenas. Z. vergl. Physiol. 21, 468-491.

Nicol, J. A. C., 1960. The biology of marine animals. Interscience publ., Inc., New York, 707 pp.

OBERTHÜR, K., 1937. Untersuchungen an Frontonia marina FABRE-Dom. aus einer Binnenland-Salzquelle unter besonderer Berïcksichtigung der pulsierenden Vakuole. Arch. Protistenk. 88, 387-420.

Panikkar, N. K., 1939. Osmotic behavior of Palaemonetes varians (Leach). Nature Lond. $144,866-867$.

- 1940. Osmotic properties of the common prawn. Nature, Lond. 145, 108.

- 1941. Osmoregulation in some palaemonid prawns. J. mar. biol. Ass. U.K. 25, 317-359.

- 1950. Physiological aspects of adaptation to estuarine conditions. Proc. Indo-Pacif. Fish. Coun. 2, 168-175.

- \& Viswanathan, R., 1948. Active regulation of chloride in Metapenaeus monoceros Fab. Nature, Lond. 161, 137-138.

Pantin, C. F. A., 1931a. The adaptation of Gunda ulvae to salinity. I. The environment. J. exp. Biol. 8, 63-72.

- 1931b. The adaptation of Gunda ulvae to salinity. 3. The electrolyte exchange. 7 . exp. Biol. 8, 82-94.

Pearse, A. S. \& Gunter, G., 1957. Salinity. Mem. geol. Soc. Am. 67, 129-157.

Potts, W. T. W. \& Parry, G., 1963. Osmotic and ionic regulation in animals. Pergamon pr., London, $402 \mathrm{pp}$.

PRECHT, $H ., 1964$. Ober die Resistenzadaptation wechselwarmer Tiere an extreme Temperaturen und ihre Ursachen. Helgol. Wiss. Meeresunters. 9, 392-411.

Pritchard, D. W., 1952. Salinity distribution and circulation in the Chesapeake Bay Estuarine System. J. mar. Res. 11, 106-123.

Prosser, C. L., 1964. Perspectives of adaptation: theoretical aspects. In: Handbook of physiology. Ed. by D. B. Dill, E. F. Adolph \& C. G. Wrlber. Am. Physiol. Soc., Wash., Sect. 4, 11-25.

- \& BRown, F. A., Jr., 1961. Comparative animal physiology. 2nd ed., Saunders, Philadelphia, Pa., 688 pp.

Provasolr, L., 1958. Nutrition and ecology of Protozoa and algae. A. Rev. Microbiol. 12, 279-308.

- Mclaughlin, J. J. A. \& Pentner, I. J., 1954. Relative and limiting concentrations of major mineral constituents for the growth of algal flagellates. Trans. N.Y. Acad. Sci. (Ser. 2), 16, 412-417.

REID, G. K., 1961. Ecology of inland waters and estuaries. Reinhold, New York, 375 pp.

Remane, A. \& SCHLIEPER, C., 1958. Die Biologie des Brackwassers. In: Die Binnengewässer. Hrsg. von A. Thienemann. Schweizerbart, Stuttgart, Bd. 22, 348 pp. 
Robertson, J. D., 1957. Osmotic and ionic regulation in aquatic invertebrates. Recent advances in invertebrate physiology. Publs Univ. Oregon, 229-246.

- 1960a. Osmotic and ionic regulation In: The physiology of Crustacea. Ed. by T. H. WATERMAN. Acad. pr., New York, Vol. 1, 317-339.

- $1960 \mathrm{~b}$. Ionic regulation in the crab Carcinus maenas (L.) in relation to the moulting cycle. Comp. Biochem. Physiol. 1, 183-2.12.

Rothschid, L. \& Barnes, H., 1953. The inorganic constituents of the sea-urching egg. J.exp. Biol. 30, 534-544.

Schlieper, C., 1929a. Über die Einwirkung niederer Salzkonzentrationen auf marine Organismen. $Z$. vergl. Physiol. 9, 478-514.

- 1929b. Neue Versuche über die Osmoregulation wasserlebender Tiere. S. B. Ges. ges. Naturw. Marburg 64, 143-156.

- 1930. Die Osmoregulation wasserlebender Tiere. Biol. Rev. 5, 309-356.

- 1935. Neuere Ergebnisse und Probleme aus dem Gebiet der Osmoregulation wasserlebender Tiere. Biol. Rev. 10, 334-360.

Schmitz, W., 1959. Zur Frage der Klassifikation der binnenländischen Brackwasser. Archo Oceanogr. Limnol. (Suppl.) 11, 179-226.

Schutz, H., 1961. Qualitative und quantitative Planktonuntersuchungen im Elbe-Astuar. Arch. Hydrobiol. (Suppl.) 26, 5-105.

Schumacher, A., 1961. Die biologischen Verhältnisse in Nebenflüssen der Unterelbe. Arch. Hydrobiol. (Suppl.) 26, 185-219.

ScotT, G. T. \& Hayward, H. R., 1953. Metabolic factors influencing the sodium and potassium distribution in Ulva lactuca. J. gen. Physiol. 36, 659-671

-, - 1954. Evidence for the presence of separate mechanisms regulating potassium and sodium distribution in Ulva lactuca. J. gen. Physiol. 37, 601-620.

-, - 1955. Sodium and potassium regulation in Ulva lactuca and Valonica macrophysa. In: Electrolytes in biological systems. Ed. by A. M. Shanes. Am. Physiol. Soc., Wash., 35-64.

SECK, C., 1958. Untersuchungen zur Frage der Ionenregulation bei in Brackwasser lebenden Evertebraten. Kieler Meeresforsch. 13, 220-243.

SHAw, J., 1960. The mechanisms of osmoregulation. In: Comparative biochemistry. Ed. by M. Florkin \& H. S. Mason. Acad. pr., New York, Vol. 2, 479-518.

SмIтH, R. I., 1955a. Comparsion of the level of chloride regulation by Nereis diversicolor in different parts of its geographical range. Biol. Bull. mar. biol. Lab., Woods Hole 109, $453-474$.

- 1955b. On the distribution of Nereis diversicolor in relation to salinity in the vicinity of Tvärminne, Finland, and the Isefjord, Denmark. Biol. Bull. mar. biol. Lab., Woods Hole 108, 326-345.

- 1955c. Salinity variation in interstitial water of sand at Kames Bay, Millport, with reference to the distribution of Nereis diversicolor. J. mar. biol. ass. U.K. 34, 33-46.

- 1956. The ecology of the Tamar estuary. 7. Observations on the interstitial salinity of intertidal muds in the estuarine habitat of Nereis diversicolor. J. mar. biol. Ass. U.K. 35, $81-104$.

- 1957. A note on the tolerance of low salinities by nereid polychaetes and its relation to temperature and reproductive habit. Annls Biol. 33, 93-107.

- 1959. Physiological and ecological problems of brackish waters. In: Marine biology. Proc. 20th Annual Biol. Coll., Oregon State College, 59-69.

Sumner, F. B., Louderback, G. D., SchmitT, W. L. \& Johnston, E. C. A., 1914. A report upon the physical conditions in San Francisco Bay. Univ. Calif. Publ. Zool. 14, 198 pp.

Topping, F. L. \& Fulier, J. L., 1942. The accommodation of some marine invertebrates to reduced osmotic pressures. Biol. Bull. mar. biol. Lab., Woods Hole 82, 372-384.

VOLLENWEIDER, R. A, 1950. Okologische Untersuchungen von planktischen Algen auf experimenteller Grundlage. Schweiz. Z. Hydrol. 12, 1-75.

WeIL, E. \& PANTrN, C. F. A., 1931. The adaption of Gunda ulvae to salinity. 2. The water exchange. J. exp. Biol. 8, 73-81.

Williams, R. B., 1962. The ecology of diatom populations in a Georgia salt marsh. Cambridge, Mass., Harvard Univ. D. Thesis. 